\title{
Dynamically complete markets under Brownian motion
}

\author{
Theodoros M. Diasakos ${ }^{1}$ (D)
}

Received: 8 July 2020 / Accepted: 25 February 2021 / Published online: 29 April 2021

(c) The Author(s) 2021

\begin{abstract}
This paper investigates how continuous-time trading renders complete a financial market in which the underlying risk process is a Brownian motion. A sufficient condition, that the instantaneous dispersion matrix of the relative dividends is non-degenerate, has been established in the literature for single-commodity, pure-exchange economies with many heterogenous agents where the securities' dividends as well as the agents' utilities and endowments include flows during the trading horizon which are analytic functions. In sharp contrast, the present analysis is based upon a different mathematical argument that assumes neither analyticity nor a particular underlying economic environment. The novelty of our approach lies in deriving closed-form expressions for the dispersion coefficients of the securities' prices. To this end, we assume only that the pricing kernels and dividends satisfy standard growth and smoothness restrictions (mild enough to allow even for options). In this sense, our sufficiency conditions apply irrespectively of preferences, endowments or other structural elements (for instance, whether or not the budget constraints include only pure exchange).
\end{abstract}

Keywords Dynamically complete markets · Endogenously complete markets · Brownian motion · Dispersion coefficients

JEL codes D53 $\cdot \mathrm{G} 10 \cdot \mathrm{G} 12$

\section{Introduction}

Whether or not a given asset market is dynamically complete is of fundamental importance in financial economics. If the pricing process of the underlying securities is dynamically complete, then options and other derivatives can be uniquely priced by arbitrage arguments and replicated by trading the underlying securities over time. In the absence of dynamic

Earlier versions had benefited from advice by Bob Anderson as well as suggestions by Philip Madan, Martin Schweizer, and two anonymous referees. The current version has benefited from suggestions by the associated editor and one anonymous referee. Many helpful comments have been received also from Paolo Ghirardato, Elisa Luciano, Miltiadis Makris, Giovanna Nicodano, Heracles Polemarchakis, Roberto Raimondo, and Chris Shannon. The usual caveat applies.

$凶$ Theodoros M. Diasakos

theodoros.diasakos@stir.ac.uk

1 Stirling Management School, University of Stirling, Stirling, Scotland 
completeness, however, this is no longer the case: no-arbitrage restrictions do not suffice to guarantee unique option prices while replication may not be possible. It is crucial therefore to be able to associate dynamic completeness with the economic primitives of a given financial environment-in a manner that remains unambiguously verifiable and holds at least generically across the space of these primitives. The present paper provides sufficient conditions for continuous-time trading to render an asset market dynamically complete when the underlying risk process is a Brownian motion.

The typical generic result for dynamic completeness in the continuous-time literature establishes the validity of the corresponding sufficient condition except for a small subset of the domain space of the primitive parameters. Yet it remains difficult, if not impossible in some cases, to establish whether the condition is valid for particular values of these parameters. Notable exceptions are the sufficiency results in Anderson and Raimondo [2], Hugonnier et al. [17], Riedel and Herzberg [28] (see also Riedel and Herzberg [27]) as well as Kramkov [19]. These studies refer to financial markets in single-commodity, pure-exchange economies with many heterogenous agents, and where all intermediate flows of utilities and endowments are analytic functions. In sharp contrast, the current exposition is based upon a different mathematical argument that assumes neither analyticity nor a particular underlying economic environment.

The novelty of our approach lies in deriving closed-form expressions for the dispersion coefficients of the relative asset prices. The respective formulae allow us in turn to establish sufficient conditions for the asset market to be dynamically complete that apply under general specifications for the pricing kernel and the securities' dividends, as long as both are continuous and satisfy standard in the literature smoothness and growth conditions. As to be expected, our sufficient conditions are non-degeneracy ones on the instantaneous dispersion matrix of primitive parameters. They do coincide with those in the aforementioned papers under analyticity. In general, however, they are stronger. This renders our analysis applicable on more general settings regarding the underlying structure for economic activity or the agents' preferences and endowments. It also sheds light on how the relation between dynamic completeness and the non-degeneracy conditions in question extends in directions that are important for applications.

The relevance of our study becomes evident when viewed in the context of general equilibrium analysis. The typical method in the literature for obtaining financial equilibria in continuous time has been to compute an Arrow-Debreu equilibrium and use the associated consumption process as pricing kernel in order to construct equilibrium prices for the traded securities. ${ }^{1}$ To ensure, however, that the starting Arrow-Debreu allocation is implementable by trading the given set of securities, their market needs to be dynamically complete-and, thus, to permit the construction of the equilibrium pricing process via a representative agent. Yet, the equilibrium pricing process is determined endogenously (via fixed-point arguments) from the model's primitives (the utility functions of the agents, their endowments and the dividend processes of the securities) and are expressed as expectations of properly discounted future payoffs. As a result, especially in economies with many heterogenous agents and apart from the extremely special cases where one can obtain sufficiently straightforward closedform solutions, verifying from the primitives that the equilibrium pricing process is indeed dynamically complete is a highly non-trivial problem, known as "endogenous completeness."

Essential progress in this problem was achieved by the aforementioned papers. The fundamental insight is that, for the asset market to be dynamically complete, it suffices that

\footnotetext{
1 See the introductory section in Anderson and Raimondo [2] for an extensive review and discussion of the relevant literature. The existence of the Arrow-Debreu equilibrium itself is due to some assumptions whose form varies in the literature.
} 
the instantaneous dispersion matrix of the relative dividends is non-degenerate (i.e., nonsingular) at some point in the space. The crucial underlying assumption is that the securities' dividends as well as the agents' utilities and endowments include flows during the trading horizon which are analytic functions (at least in time). By relaxing this restriction, the present study complements these papers shedding light on the intuition behind their fundamental underpinnings.

For example, when the securities' dividends comprise only lump sums at the terminal date of the trading horizon, our sufficiency condition is exactly the same as above-even though we do not assume analyticity. This remains the case when the dividends include flows during the trading horizon, as long as a collection of simple options is available for trading. More tellingly perhaps, when the securities' dividends comprise only flows during the trading horizon (a common setup especially in applied finance models), our sufficiency condition requires that the instantaneous dispersion matrix of the relative nominal dividends is nondegenerate everywhere in the space. This highlights the implications of assuming analyticity. Without it we end up with a sufficiency condition that is, on the one hand, more burdensome to verify. On the other hand, it does ensure that the instantaneous dispersion matrix of the relative securities' prices is non-degenerate everywhere, not almost everywhere, in the space.

The balance of the paper is organized as follows. The next section introduces the theoretical structure under investigation. Section 3 presents our results and Sect. 4 interprets them in the context of the pertinent literature. Section 5 concludes while the "Appendix" presents the supporting technical material and results.

\section{Theoretical framework}

Consider a financial market where the trading horizon is $\mathcal{T}=[0, T]$ for some $T \in \mathbb{R}_{++}$ or $\mathcal{T}=\mathbb{R}_{+}$while the informational structure is given by a $K$-dimensional $(K \in \mathbb{N} \backslash\{0\})$ standard Brownian process, defined on a complete probability space $(\Omega, \mathcal{F}, \pi)$ and denoted by $\beta: \Omega \times \mathcal{T} \rightarrow \mathbb{R}^{K}$ (or $\beta_{k}: \Omega \times \mathcal{T} \rightarrow \mathbb{R}$ with $k \in \mathcal{K}:=\{1, \ldots, K\}$ for the typical dimension). As usual, the process is meant to fully describe the exogenous financial risk in the sense that the collection of the sample paths $\{\beta(\omega, t): t \in \mathcal{T}\}_{\omega \in \Omega}$ specifies all the distinguishable events.

The underlying risk process being a Brownian motion, a necessary condition for any securities market to be dynamically complete is that the number of securities exceeds that of independent Brownian motions by at least one (i.e., that the market is potentially complete). In what follows the trading structure will consist of $K+1$ dividend-paying securities, indexed by $j \in \mathcal{K} \cup\{0\}$ and traded continuously over $\mathcal{T}$. These are real assets in the sense that their dividends are in units of an underlying physical commodity. The dividends will take one or both of two forms, a flow and a lump sum. Specifically, letting $\mathcal{I}: \Omega \times \mathcal{T} \rightarrow \mathcal{T} \times \mathbb{R}^{K}$ denote the process $\{t, \beta(\omega, t)\}_{(\omega, t) \in \Omega \times \mathcal{T}}$, we consider the functions $G_{j}, g_{j}: \mathcal{T} \times \mathbb{R}^{K} \rightarrow \mathbb{R}_{+}$ and that the typical security pays the dividend flow $g_{j}(\mathcal{I}(\omega, \cdot))$ along the Brownian path $\{\beta(\omega, t): t \in \mathcal{T}\}$ and/or the lump sum $G_{j}(\mathcal{I}(\omega, T))$ on the terminal date (if the time horizon is finite: $\mathcal{T}=[0, T]$ for some $\left.T \in \mathbb{R}_{++}\right)$. The supports $S_{G_{j}}:=\left\{\beta \in \mathbb{R}^{K}: G_{j}(T, \beta)>0\right\}$ and $S_{g_{j}}:=\left\{(t, \beta) \in \mathcal{T} \times \mathbb{R}^{K}: g_{j}(t, \beta)>0\right\}$ will be taken as open and connected. Letting moreover $D_{k}:=\partial / \partial \beta_{k}$ denote the instantaneous dispersion operator, we will also assume that, for any $k \in \mathcal{K}$, the functions $D_{k} G_{j}(T, \cdot)$ and $D_{k} g_{j}(\cdot)$ are well defined and continuous on $S_{G_{j}}$ and $S_{g_{j}}$, respectively. 
Given that trading occurs over a time interval while the informational structure is driven by a Brownian motion, well-known no-arbitrage conditions ensure that the securities' prices are the current expectations of their future dividends valued at some pricing kernel, a strictly positive one-dimensional Itô process. In what follows, we take the pricing kernel to be given by the functions $M, m: \mathcal{T} \times \mathbb{R}^{K} \rightarrow \mathbb{R}_{++}$which are continuously differentiable on $\operatorname{int}(\mathcal{T}) \times \mathbb{R}^{K}$. In addition, we assume that the functions $M_{j}(\cdot):=\left(M G_{j}\right)(\cdot)$ and $m_{j}(\cdot):=\left(m g_{j}\right)(\cdot)$ satisfy appropriate growth conditions (see assumptions A1-A2 below) for the price of the typical security to be written as follows

$$
P_{j}(t, \beta)=\mathbb{E}_{\pi}\left[\mathbf{1}_{\mathcal{T} \neq \mathbb{R}_{+}} \times \frac{M_{j}\left(T, \beta_{T}\right)}{m(t, \beta)}+\int_{\mathcal{T} \backslash(0, t)} \frac{m_{j}\left(s, \beta_{s}\right)}{m(t, \beta)} \mathrm{d} s \mid \beta_{t}=\beta\right] \quad(t, \beta) \in \mathcal{T} \times \mathbb{R}^{K}
$$

All prices above being strictly positive, any one can be used as deflator. Without loss of generality, therefore, we may deflate all prices by that of the zeroth security and restrict attention to the relative pricing process $p_{j}(\cdot):=P_{j}(\cdot) / P_{0}(\cdot), j \in \mathcal{K}$. This normalization renders the zeroth security instantaneously risk free (for its price remains constant at 1 ) so that the strategy of buying and holding it becomes a (trivial) money-market account. As a result, the financial market under consideration is dynamically complete if and only if the (Jacobian) matrix of the instantaneous dispersions of the relative prices, $J_{p}(\cdot):=\left[D_{k} p_{n}(\cdot)\right]_{(n, k) \in \mathcal{K} \times \mathcal{K}}$, is non-singular almost everywhere on $\operatorname{int}(\mathcal{T}) \times \mathbb{R}^{K} .^{2}$

In what follows, we seek to establish sufficient conditions for the Jacobian matrix to have the desired property. To this end we observe that, since $p_{n}(\cdot)=\left(m P_{n}\right)(\cdot) /\left(m P_{0}\right)(\cdot)$ using the nominal prices $\left(m P_{j}\right)(\cdot):=m(\cdot) P_{j}(\cdot)$ for $j \in \mathcal{K} \cup\{0\}$, an equivalent statement of our aim is to ensure that $\left[\left(m P_{0}\right)(\cdot) D_{k}\left(m P_{n}\right)(\cdot)-\left(m P_{n}\right)(\cdot) D_{k}\left(m P_{0}\right)(\cdot)\right]_{(n, k) \in \mathcal{K} \times \mathcal{K}}$ remains non-singular almost everywhere on $\operatorname{int}(\mathcal{T}) \times \mathbb{R}^{K}$. As it turns out, the latter matrix is well defined on int $(\mathcal{T}) \times \mathbb{R}^{K}$ under the following conditions. ${ }^{3}$

A 1 Let $\mathcal{T}=[0, T]$ for some $T \in \mathbb{R}_{++}$. There exist constants $\left(C_{0}, r_{0}\right) \in \mathbb{R}_{++} \times\left(0, \frac{1}{2 T}\right)$ such that

$$
\begin{aligned}
& \text { (i) } M_{j}(T, \mathbf{x})+\left|D_{k} M_{j}(T, \mathbf{x})\right| \leq C_{0} e^{r_{0}|\mathbf{x}|^{2}} \forall \mathbf{x} \in S_{G_{j}} \\
& \text { (ii). } m_{j}(s, \mathbf{x})+\left|D_{k} m_{j}(s, \mathbf{x})\right| \leq C_{0} e^{r_{0}|\mathbf{x}|^{2}} \forall(s, \mathbf{x}) \in S_{g_{j}}
\end{aligned}
$$

for all $(j, k) \in \mathcal{K} \cup\{0\} \times \mathcal{K}$.

A 2 Let $\mathcal{T}=\mathbb{R}_{+}$. For every constant $r>0$ there exists a constant $C>0$ such that

$$
m_{j}(s, \mathbf{x})+\left|D_{k} m_{j}(s, \mathbf{x})\right| \leq C e^{r|\mathbf{x}|^{2}} \quad \forall(s, \mathbf{x}) \in S_{g_{j}}
$$

for all $(j, k) \in \mathcal{K} \cup\{0\} \times \mathcal{K}$.

\footnotetext{
2 The claims in this paragraph are based on well-known concepts and results. See, for example, Chapters 5A-C, 6I, and 6K in Duffie [11] as well as Sections 4.1-4.4 and Theorem 5.6 in Nielsen [24]. Observe also that, as usual, $\beta_{t}$ denotes a (random) value of the mapping $t \mapsto\{\beta(\omega, t)\}_{\omega \in \Omega}$.

${ }^{3}$ As usual, $|\cdot|$ denotes the Euclidean norm of a vector or the determinant of a (square) matrix. Notice also that the conditions A1-A2 are trivially satisfied on $\{T\} \times \operatorname{int}\left(\mathbb{R}^{K} \backslash S_{G_{j}}\right)$ and $\operatorname{int}\left(\mathcal{T} \times \mathbb{R}^{K} \backslash S_{g_{j}}\right)$ when these are non-empty (for instance, when the security is a European option-see Claim 3.2 below). Indeed, we have $G_{j}(T, \cdot)=0=D_{k} G_{j}(T, \cdot)$ on the former and $g_{j}(\cdot)=0=D_{k} g_{j}(\cdot)$ on the latter.
} 


\section{Analysis}

Starting with the first term in (1), and letting $\mathcal{T}:=[0, T]$ for some $T \in \mathbb{R}_{++}$, recall that the increment $\beta(\cdot, T)-\beta(\cdot, t)$ is independent of $\mathcal{F}_{t}$ and distributed $\mathcal{N}\left(\mathbf{0}^{K},(T-t) \mathrm{I}_{K}\right)$ over $\Omega$. Hence, the term in question can be written as

$$
P_{1 j}(t, \beta):=\int_{\mathbb{R}^{K}} \frac{M_{j}(\beta+\sqrt{T-t} \mathbf{x})}{m(t, \beta)} \phi(\mathbf{x}) \mathrm{d} \mathbf{x}, \quad(t, \beta) \in[0, T) \times \mathbb{R}^{K}, \quad j \in \mathcal{K} \cup\{0\}
$$

where $\phi(\cdot)$ denotes the $K$-dimensional standard-normal pdf.

Under Assumption A1(i), the pricing function in (2) above is well defined. And so is the dispersion with respect to the typical Brownian dimension of its nominal version. Specifically, we have

$$
D_{k}\left(m P_{1 j}\right)(t, \beta)=\mathbb{E}_{\mathbf{x}}\left[D_{k} M_{j}(T, \beta+\sqrt{T-t} \mathbf{x})\right],(t, \beta) \in[0, T) \times \mathbb{R}^{K}, \quad j \in \mathcal{K} \cup\{0\}
$$

where $\mathbf{x} \sim \mathcal{N}\left(\mathbf{0}^{K}, \mathrm{I}_{K}\right)$. For $t \rightarrow T$, moreover, the continuity in $t$ commutes inside the expectation operator above. As a result, letting $p_{1 n}(\cdot):=P_{1 n}(\cdot) / P_{10}(\cdot)$ and $G_{n / 0}(\cdot):=$ $\mathbf{1}_{S_{G_{0}}} \times G_{n}(\cdot) / G_{0}(\cdot)$ denote, respectively, the relative prices and terminal dividends, the (Jacobian) dispersion matrix of the relative prices, $J_{p_{1}}(\mathcal{I}(\omega, t))$, approaches that of the relative terminal dividends

$$
J_{G}(\mathcal{I}(\omega, T)):=\left[D_{k} G_{n / 0}(\mathcal{I}(\omega, T))\right]_{(n, k) \in \mathcal{K} \times \mathcal{K}}
$$

almost everywhere in $\left\{\omega \in \Omega: \beta(\omega, T) \in S_{G_{0}}\right\} .4$

The latter observation leads to a sufficient condition for the financial market to be dynamically complete in the case where the time horizon is finite and the securities pay only lump-sum dividends on the terminal date.

Theorem 3.1 Let $\mathcal{T}=[0, T]$ for some $T \in \mathbb{R}_{++}$and suppose that the price process is given by (2) with $A 1(i)$ satisfied. Then $J_{p_{1}}(\cdot)$ has full rank almost everywhere on $(0, T) \times \mathbb{R}^{K}$ if $J_{G}(\cdot)$ is non-singular at some $\beta_{T} \in S_{G_{0}}$.

Proof Our argument will be based upon the fact that, even though we have not required analyticity anywhere above, the entries of the dispersion matrix $J_{p}(\cdot)$ are analytic on $(0, T) \times \mathbb{R}^{K}$ under Assumption A1(i). This is because, for any $(j, k) \in \mathcal{K} \cup\{0\} \times \mathcal{K}$, the functions $\left(m P_{j}\right)(\cdot)$ and $D_{k}\left(m P_{j}\right)(\cdot)$ are analytic on $(0, T) \times \mathbb{R}^{K}$. For the former function, its analyticity follows immediately from Lemma A.7 in the "Appendix". For the latter function, since $\left|x_{k}\right| \leq|\mathbf{x}|<e^{|\mathbf{x}|}<1+e^{|\mathbf{x}|}$ everywhere on $\mathbb{R}^{K}$, by Lemma A.1 in the "Appendix", there exist constants $(C, r) \in \mathbb{R}_{++} \times\left(0, \frac{1}{2 T}-r_{j}\right)$ such that $\left|x_{k}\right| \leq C e^{r|\mathbf{x}|^{2}}$ for any $\mathbf{x} \in \mathbb{R}^{K}$. Under Assumption A1(i), therefore, we have $\left|x_{k} M_{j}(T, \mathbf{x})\right| \leq C_{0} C e^{\left(r_{0}+r\right)|\mathbf{x}|^{2}}$ anywhere on $\mathbb{R}^{K}$, with $r_{0}+r \in\left(0, \frac{1}{2 T}\right)$. By Lemma A.7 in the "Appendix" then

$$
\begin{aligned}
\left(m P_{j}\right)(t, \beta) \beta_{k}+(T-t) D_{k}\left(m P_{j}\right)(t, \beta) & =\mathbb{E}_{\mathbf{x}}\left[\left(\beta_{k}+\sqrt{T-t} x_{k}\right) M_{j}(T, \beta+\sqrt{T-t} \mathbf{x})\right] \\
& =\mathbb{E}\left[\beta_{k T} M_{j}\left(T, \beta_{T}\right) \mid \beta_{t}=\beta\right]
\end{aligned}
$$

\footnotetext{
4 The claims in the main text above are based on supporting results in the "Appendix". Specifically, that $\left(m P_{1 j}\right)(\cdot)$ and $D_{k}\left(m P_{1 j}\right)(\cdot)$ are both well defined with the latter given by (3) follow from Lemma A.5. Moreover, that continuity with respect to time commutes inside the expectations in (2) and (3) follows from Lemmas A.6-A.3. Finally, that $J_{p_{1}}(\mathcal{I}(\omega, t))$ approaches $J_{G}(\mathcal{I}(\omega, T))$ is due to Proposition A.1.
} 
is an analytic function of $(t, \beta)$ on $(0, T) \times \mathbb{R}^{K}$. That $D_{k}\left(m P_{j}\right)(\cdot)$ itself is analytic on $(0, T) \times \mathbb{R}^{K}$ follows now from the fact that the sum, product and ratio of two real analytic functions are also analytic (see Propositions 1.1.7 and 1.1.12 in Krantz and Parks [20]).

Suppose now that $\left|J_{G}\left(T, \beta^{0}\right)\right| \neq 0$ for some $\beta^{0} \in S_{G_{0}}$, and take $\omega_{0} \in \Omega$ such that $\beta^{0}=\beta\left(\omega_{0}, T\right)$. By continuity, there exists $\delta_{0}>0$ such that $\left|J_{G}(T, \beta)\right| \neq 0$ for any $\beta \in \mathcal{B}_{\beta^{0}}\left(\delta_{0}\right)$. Take also $\omega_{1} \in \Omega$ such that $\lim _{t \rightarrow T} J_{p}\left(t, \beta\left(\omega_{1}, t\right)\right)=J_{G}\left(T, \beta^{1}\right)$ where $\beta^{1}:=\beta\left(\omega_{1}, T\right) \in \mathcal{B}_{\beta^{0}}\left(\delta_{0}\right)$. By Proposition A.1 in the "Appendix", and as the determinant of a matrix is a continuous operator, we have

$$
\lim _{t \rightarrow T}\left|J_{p}\left(t, \beta\left(\omega_{1}, t\right)\right)\right|=\left|J_{G}\left(T, \beta^{1}\right)\right| \neq 0
$$

There exist therefore $\left(\delta_{1}, t_{1}\right) \in \mathbb{R}_{++} \times(0, T)$ such that

$$
\left|J_{p}(s, \beta)\right| \neq 0 \quad \forall(s, \beta) \in\left(t_{1}, T\right) \times \mathcal{B}_{\beta^{1}}\left(\delta_{1}\right)
$$

As established above, though, the entries of $J_{p}(\cdot)$ are analytic on $(0, T) \times \mathbb{R}^{K}$. The determinant of a matrix involving nothing but the operations of product and sum on its entries, $\left|J_{p}(\cdot)\right|$ is also analytic on $(0, T) \times \mathbb{R}^{K}$. But then (4) necessitates that $\left|J_{p}(\cdot)\right| \neq 0$ almost everywhere on $(0, T) \times \mathbb{R}^{K}$.

Our argument for establishing Theorem 3.1 hinges crucially upon the fact that the functions $\left(m P_{j}\right)(\cdot)$ and $D_{k}\left(m P_{j}\right)(\cdot)$ are both analytic on $(0, T) \times \mathbb{R}^{K}$, even though $M_{j}(\cdot)$ itself is not assumed to be analytic. Unfortunately, this fact does not obtain for the respective functions when it comes to the second term in (1). The analysis of the pricing process for the flow divedends requires its own approach.

Under Assumption A1(ii), the pricing function

$$
P_{2 j}(t, \beta):=\mathbb{E}_{\pi}\left[\int_{\mathcal{T} \backslash(0, t)} \frac{m_{j}\left(s, \beta_{s}\right)}{m(t, \beta)} \mathrm{d} s \mid \beta_{t}=\beta\right] \quad(t, \beta) \in \mathcal{T} \times \mathbb{R}^{K} \quad j \in \mathcal{K} \cup\{0\}
$$

but also the dispersion with respect to the typical Brownian dimension of its nominal version are both well defined. Specifically, we have ${ }^{6}$

$$
\begin{aligned}
\left(m P_{2 j}\right)(t, \beta) & =\int_{t}^{T} \mathbb{E}_{\mathbf{x}}\left[m_{j}(s, \beta+\sqrt{s-t} \mathbf{x})\right] \mathrm{d} s \\
D_{k}\left(m P_{2 j}\right)(t, \beta) & =\int_{t}^{T} \mathbb{E}_{\mathbf{x}}\left[D_{k} m_{j}(s, \beta+\sqrt{s-t} \mathbf{x})\right] \mathrm{d} s \\
& =\int_{t}^{T} \mathbb{E}_{\pi}\left[D_{k} m_{j}\left(s, \beta_{s}\right) \mid \beta_{\mathbf{t}}=\beta\right] \mathrm{d} s
\end{aligned}
$$

And for the case $\mathcal{T}=\mathbb{R}_{+}$, note that the respective right-hand sides of (6)-(7) above remain well defined at all $T \in \mathbb{R}_{++}$under condition A2. Hence, their limiting versions are given by

$$
\begin{gathered}
\left(m P_{2 j}\right)(t, \beta)=\int_{t}^{\infty} \mathbb{E}_{\pi}\left[m_{j}\left(s, \beta_{s}\right) \mid \beta_{t}=\beta\right] \mathrm{d} s \\
D_{k}\left(m P_{2 j}\right)(t, \beta)=\int_{t}^{\infty} \mathbb{E}_{\pi}\left[D_{k} m_{j}\left(s, \beta_{s}\right) \mid \beta_{t}=\beta\right] \mathrm{d} s
\end{gathered}
$$

\footnotetext{
5 A real analytic function defined on an open and convex subset of $\mathbb{R}^{n}(n \in \mathbb{N} \backslash\{0\})$ is either zero everywhere on its domain or non-zero almost everywhere (see Theorem B.3 in Anderson and Raimondo [2]).

6 The claims made above with respect to the expressions in (6)-(8) are supported by the results and discussion in Sect. B in the "Appendix". It is also noteworthy that (8) can be shown to follow directly from (6) by deploying though Malliavin calculus—see for instance Appendix A in Detemple and Zapatero [10].
} 
Letting then $p_{2 n}(\cdot):=P_{2 n}(\cdot) / P_{20}(\cdot)$, the above findings can be summarized as follows.

Claim 3.1 Let the price process be given by (5) with $\mathcal{T}=[0, T]$ for some $T \in \mathbb{R}_{++}$or $\mathcal{T}=\mathbb{R}_{++}$and $A 1($ ii) or $A 2$, respectively, satisfied. Then

$$
D_{k}\left(m P_{2 j}\right)(t, \beta)=\int_{\mathcal{T} \backslash(0, t)} \mathbb{E}_{\pi}\left[D_{k} m_{j}\left(s, \beta_{s}\right) \mid \beta_{t}=\beta\right] d s
$$

and thus

$$
\begin{aligned}
& \left(m P_{20}\right)(t, \beta)^{2} D_{k} p_{2 n}(t, \beta) \\
& =\int_{\mathcal{T} \backslash(0, t)} \int_{\mathcal{T} \backslash(0, t)} \mathbb{E}_{\pi}\left[m_{0}\left(\tau, \beta_{\tau}\right) D_{k} m_{n}\left(s, \beta_{s}\right)-m_{n}\left(s, \beta_{s}\right) D_{k} m_{0}\left(\tau, \beta_{\tau}\right) \mid \beta_{t}=\beta\right] d s d \tau
\end{aligned}
$$

at any $(t, \beta) \in \operatorname{int}(\mathcal{T}) \times \mathbb{R}^{K}$ and for any $(j, n, k) \in \mathcal{K} \cup\{0\} \times \mathcal{K} \times \mathcal{K}$.

The preceding relations shed light as to why our proof for Theorem 3.1 does not extend to the case of flow dividends. For it would establish here that the intergrands in (6)-(7) are analytic in $(t, \beta)$ for each given $s$. However, unless one assumes in addition that these integrands are also analytic in $s$, it does not follow that $\left(m P_{2 j}\right)(\cdot)$ and $D_{k}\left(m P_{2 j}\right)(\cdot)$ are necessarily analytic on int $(\mathcal{T}) \times \mathbb{R}^{K}$.

This notwithstanding, the explicit expressions for the diffusion coefficients in Claim 3.1 allow a more direct approach. To this end, we define the collection $\left\{m_{n / 0}^{*}\right\}_{n \in \mathcal{K}}$ of the vectorvalued functions $m_{n / 0}^{*}: S_{g_{n}} \times S_{g_{0}} \rightarrow \mathbb{R}^{K}$ given by

$$
m_{n / 0}^{*}((s, \mathbf{x}),(\tau, \mathbf{y})):=\left(D_{1}\left(\frac{m_{n}(s, \mathbf{x})}{m_{0}(\tau, \mathbf{y})}\right), \ldots, D_{K}\left(\frac{m_{n}(s, \mathbf{x})}{m_{0}(\tau, \mathbf{y})}\right)\right)^{\top}
$$

and a stronger than the standard notion of matrix non-singularity.

Definition 1 Taking $L \in \mathbb{N} \backslash\{0\}$, let $\left\{f_{k}\right\}_{k \in \mathcal{K}}$ be a collection of functions $f_{k}: \mathbb{R}^{L} \supseteq X_{k} \rightarrow$ $\mathbb{R}^{K}$. We will say that $\left\{f_{k}\right\}_{k \in \mathcal{K}}$ satisfies strong non-singularity on $X:=\prod_{k \in \mathcal{K}} X_{k}$ if there exists no $\left(\mathbf{x}^{1}, \ldots, \mathbf{x}^{K}\right) \in X$ rendering the matrix

$$
\left[f_{1}\left(\mathbf{x}^{1}\right) \cdots f_{K}\left(\mathbf{x}^{K}\right)\right]
$$

singular.

This notion of matrix non-singularity leads to a sufficient condition for the financial market to be dynamically complete in the case where the securities' dividends comprise only flows during the time horizon. Specifically, letting $J_{p_{2}}(\cdot):=\left[D_{k} p_{2 n}(\cdot)\right]_{(n, k) \in \mathcal{K} \times \mathcal{K}}$, we have the following.

Theorem 3.2 Let the price process be given by (5) with $\mathcal{T}=[0, T]$ for some $T \in \mathbb{R}_{++}$or $\mathcal{T}=\mathbb{R}_{++}$and $A 1(i i)$ or $A 2$, respectively, satisfied. Then $J_{p_{2}}(\cdot)$ has full rank everywhere on $\operatorname{int}(\mathcal{T}) \times \mathbb{R}^{K}$ if the collection $\left\{m_{n / 0}^{*}\right\}_{n \in \mathcal{K}}$ satisfies strong non-singularity on $\prod_{n \in \mathcal{K}} S_{g_{n}} \times S_{g_{0}}$. 
Proof Take arbitrary $(t, \beta) \in \operatorname{int}(\mathcal{T}) \times \mathbb{R}^{K}$ and $\mathbf{v} \in \mathbb{R}^{K} \backslash\left\{0^{K}\right\}$. By Claim 3.1, the typical entry in the matrix $\left(m P_{0}\right)(t, \beta)^{2} J_{p}(t, \beta)$ is given as

$$
\begin{aligned}
& \left(m P_{0}\right)(t, \beta)^{2} D_{k} p_{n}(t, \beta) \\
& =\int_{\mathcal{T} \backslash(0, t)} \int_{\mathcal{T} \backslash(0, t)} \mathbb{E}_{\pi}\left[m_{0}\left(\tau, \beta_{\tau}\right) D_{k} m_{n}\left(s, \beta_{s}\right)-m_{n}\left(s, \beta_{s}\right) D_{k} m_{0}\left(\tau, \beta_{\tau}\right) \mid \beta_{t}=\beta\right] \mathrm{d} s \mathrm{~d} \tau \\
& =\int_{S_{g_{n}}} \int_{S_{g_{0}}} \mathbb{E}_{\pi}\left[m_{0}\left(\tau, \beta_{\tau}\right) D_{k} m_{n}\left(s, \beta_{s}\right)-m_{n}\left(s, \beta_{s}\right) D_{k} m_{0}\left(\tau, \beta_{\tau}\right) \mid \beta_{t}=\beta\right] \mathrm{d} s \mathrm{~d} \tau \\
& =\int_{S_{g_{n}}} \int_{S_{g_{0}}} \mathbb{E}_{\pi}\left[m_{0}\left(\tau, \beta_{\tau}\right)^{2} D_{k}\left(\frac{m_{n}\left(s, \beta_{s}\right)}{m_{0}\left(\tau, \beta_{\tau}\right)}\right) \mid \beta_{t}=\beta\right] \mathrm{d} s \mathrm{~d} \tau
\end{aligned}
$$

Suppose now that the collection $\left\{m_{n / 0}^{*}\right\}_{n \in \mathcal{K}}$ satisfies strong non-singularity on $\prod_{n \in \mathcal{K}} S_{g_{n}} \times$ $S_{g_{0}}$. There exists then no $\left(\left(s_{1}, \beta_{s_{1}}\right), \ldots,\left(s_{K}, \beta_{S_{K}}\right)\right) \in \prod_{n \in \mathcal{K}} S_{g_{n}}$ and no $\left(\left(\tau_{1}, \beta_{\tau_{1}}\right), \ldots\right.$, $\left.\left(\tau_{K}, \beta_{\tau_{K}}\right)\right) \in S_{g_{0}}^{K}$ rendering the matrix

$$
\left[m_{1 / 0}^{*}\left(\left(s_{1}, \beta_{s_{1}}\right),\left(\tau_{1}, \beta_{\tau_{1}}\right)\right) \cdots m_{K / 0}^{*}\left(\left(s_{K}, \beta_{s_{K}}\right),\left(\tau_{K}, \beta_{\tau_{K}}\right)\right)\right]
$$

singular. It is trivial to check that this property requires in turn the existence of some $n \in \mathcal{K}$ such that

$$
m_{n / 0}^{*}\left(\left(s, \beta_{s}\right),\left(\tau, \beta_{\tau}\right)\right)^{\top} \mathbf{v} \neq 0 \quad \forall\left(\left(s, \beta_{s}\right),\left(\tau, \beta_{\tau}\right)\right) \in S_{g_{n}} \times S_{g_{0}}
$$

As $S_{g_{n}} \times S_{g_{0}}$ though is connected, by continuity this can be only if $m_{n / 0}^{*}(\cdot)$ maintains the same sign everywhere on $S_{g_{n}} \times S_{g_{0}}$. Without loss of generality, therefore, we may let $m_{n / 0}^{*}(\cdot)>0$ everywhere on $S_{g_{n}} \times S_{g_{0}}$. But this implies in turn that

$$
\begin{aligned}
& \left(m P_{0}\right)(t, \beta)^{2} \sum_{k \in \mathcal{K}} v_{k} D_{k} p_{n}(t, \beta) \\
& =\int_{S_{g_{n}}} \int_{S_{g_{0}}} \mathbb{E}_{\pi}\left[m_{0}\left(\tau, \beta_{\tau}\right)^{2} \sum_{k \in \mathcal{K}} v_{k} D_{k}\left(\frac{m_{n}\left(s, \beta_{s}\right)}{m_{0}\left(\tau, \beta_{\tau}\right)}\right) \mid \beta_{t}=\beta\right] \mathrm{d} s \mathrm{~d} \tau \\
& =\int_{S_{g_{n}}} \int_{S_{g_{0}}} \mathbb{E}_{\pi}\left[m_{0}\left(\tau, \beta_{\tau}\right)^{2} m_{n / 0}^{*}\left(\left(s, \beta_{s}\right),\left(\tau, \beta_{\tau}\right)\right)^{\top} \mathbf{v}\right] \mathrm{d} s \mathrm{~d} \tau>0
\end{aligned}
$$

That is, $J_{p}(t, \beta) \mathbf{v} \neq 0^{K}$ and the claim follows.

Turning finally to the case when both terms on the right-hand side of (1) apply, the pricing process $P_{j}(\cdot)=P_{1 j}(\cdot)+P_{2 j}(\cdot)$ produces even more complex dynamics for the relative prices. Nonetheless, the preceding analysis provides again a sufficient condition for the financial market to be dynamically complete when the securities pay both dividend flows and lump sums - as long as the market includes a zero-coupon bond maturing at $T$ as well as, for each $j \in \mathcal{K}$, a European call option maturing at $T$ along with its equivalent put.

Claim 3.2 Let $\mathcal{T}=[0, T]$ for some $T \in \mathbb{R}_{++}$and the price process be given by (1) with Al(i) satisfied. Suppose also that the zeroth security is a zero-coupon bond maturing at $T$ (i.e., $G_{0}(T, \cdot)=1$ and $g_{0}(\cdot)=0$ ) while, for each $j \in \mathcal{K}$, a European call maturing at $T$ (with strike price some $X_{j}>0$ ) along with its equivalent put are available for trading. Then $J_{p}(\cdot)$ has full rank almost everywhere on $[0, T) \times \mathbb{R}^{K}$ if $J_{G}(T, \cdot)$ is non-singular at some $\beta \in S_{G_{0}}$. 
Proof Observe first that, for any given $\beta \in \mathbb{R}^{K}$, we have that $\lim _{t \rightarrow T}\left(m P_{2 j}\right)(t, \beta)=0$. As a result, we have that $\lim _{t \rightarrow T}\left(m P_{j}\right)(\mathcal{I}(\omega, t))=\lim _{t \rightarrow T}\left(m P_{1 j}\right)(\mathcal{I}(\omega, t))=M_{j}(\mathcal{I}(\omega, T))$ almost everywhere on $\Omega$. Clearly, at any $(t, \beta) \in[0, T) \times \mathbb{R}^{K}$, the nominal price of a European call on the $j$ th security with maturity date $T$ and strike price $X_{j}$ and that of its equivalent put are given, respectively, by

$$
\begin{aligned}
& \left(m P_{j}^{C}\right)(t, \beta)=\int_{V_{X_{j}}(t, \beta)} M(T, \beta+\sqrt{T-t} \mathbf{x})\left(G_{j}(T, \beta+\sqrt{T-t} \mathbf{x})-X_{j}\right) \phi(\mathbf{x}) \mathrm{d} \mathbf{x} \\
& \left(m P_{j}^{P}\right)(t, \beta)=\int_{\mathbb{R}^{K} \backslash V_{X_{j}}(t, \beta)} M(T, \beta+\sqrt{T-t} \mathbf{x})\left(X_{j}-G_{j}(T, \beta+\sqrt{T-t \mathbf{x}})\right) \phi(\mathbf{x}) \mathrm{d} \mathbf{x}
\end{aligned}
$$

where

$$
V_{X_{j}}(t, \beta):=\left\{\mathbf{x} \in \mathbb{R}^{K}: G_{j}(T, \beta+\sqrt{T-t} \mathbf{x}) \geq X_{j}\right\}
$$

This implies of course the put-call parity

$$
P_{j}^{C}(t, \beta)-P_{j}^{P}(t, \beta)=P_{1 j}(t, \beta)-X_{j} B_{0}(t, \beta) \quad j \in \mathcal{K} \cup\{0\}
$$

where

$$
B_{0}(t, \beta):=\mathbb{E}_{\mathbf{x}}[M(T, \beta+\sqrt{T-t} \mathbf{x})] / m(t, \beta)=P_{10}(t, \beta)=P_{0}(t, \beta)
$$

is the current price of the zero-coupon bond.

For each $j \in \mathcal{K}$ consider the portfolio that consists of being long one unit on the corresponding call, short one unit on the equivalent put, and long $K_{j}$ units on the zero-coupon bond. The value of this portfolio being $P_{1 j}(\cdot)$, the Jacobian of the relative prices for the overall $K$ such portfolios is given by $J_{G}(\cdot)$. The claim now follows from Theorem 3.1.

\subsection{Dynamics with money-market accounts}

The preceding analysis applies also when the zeroth security is a money-market account. To see this, let $\left\{r_{t}: t \in \mathcal{T}\right\}$ be an instantaneously riskless rate process and $P_{0 t}:=$ $P_{0} \exp \left(\int_{0}^{t} r_{s} \mathrm{~d} s\right)$ for some initial value $P_{0}>0 .^{7}$ As $D_{k} P_{0 t}=0$ for any $k \in \mathcal{K}$, we now

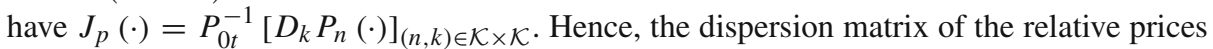
is non-singular if and only if so is the dispersion matrix of the absolute prices.

With respect to the case where the securities' dividends comprise only flows during the time horizon, the latter equivalence means that the argument establishing Theorem 3.2 above remains valid. For the equivalence ensures that, as far as dynamic completeness is concerned, there is no loss of generality if we consider the (counterfactual) market in which the securities' prices are normalized using an annuity (i.e., a security with dividend $G_{0}(\cdot)=0$ and $g_{0}(\cdot)=$ 1) instead of the money-market account as the numeraire. ${ }^{8}$

\footnotetext{
7 The construction of the riskless-rate process derives from the Radon-Nikodym derivative of an equivalentto- $\pi$ martingale measure (see, for instance, Chapter 6 and Appendix C in Duffie [11]). That the latter is well defined here follows from the fact that $m(T, \cdot)$ satisfies assumption A1(ii).

8 Notice that, in the presence of a money-market account, the riskless-rate process and the pricing kernel are related according to the dynamics $r_{t} \mathrm{~d} t=-\mathbb{E}_{\pi}\left[\mathrm{d} m_{t} \mid \mathcal{F}_{t}\right] / m_{t}$. As a result, the instantaneous change in the annuity price, $P_{A t}=\mathbb{E}\left[\int_{\mathcal{T} \backslash[0, t)} m(s) \mathrm{d} s \mid \mathcal{F}_{t}\right] / m_{t}$, matches exactly the instantaneous rate of return on the money-market account.
} 
Corollary 3.1 Let the price process be given by (5) with $\mathcal{T}=[0, T]$ for some $T \in \mathbb{R}_{++}$or $\mathcal{T}=\mathbb{R}_{++}$and A1(ii) or A2, respectively, satisfied. Then $\left[D_{k} P_{2 n}(\cdot)\right]_{(n, k) \in \mathcal{K} \times \mathcal{K}}$ has full rank everywhere on $\operatorname{int}(\mathcal{T}) \times \mathbb{R}^{K}$ if the collection $\left\{m_{n}^{*}\right\}_{n \in \mathcal{K}}$ of the functions $m_{n}^{*}: S_{g_{n}} \times \mathbb{R}^{K} \rightarrow \mathbb{R}^{K}$ given by

$$
m_{n}^{*}((s, \mathbf{x}),(\tau, \mathbf{y})):=\left(D_{1}\left(\frac{m_{n}(s, \mathbf{x})}{m(\tau, \mathbf{y})}\right), \ldots, D_{K}\left(\frac{m_{n}(s, \mathbf{x})}{m(\tau, \mathbf{y})}\right)\right)^{\top}, \quad n \in \mathcal{K}
$$

satisfies strong non-singularity on $\prod_{n \in \mathcal{K}} S_{g_{n}} \times \mathbb{R}^{K}$.

Proof Take arbitrary $(t, \beta) \in \operatorname{int}(\mathcal{T}) \times \mathbb{R}^{K}$ and $(n, k) \in \mathcal{K} \times \mathcal{K}$. By rearranging terms in the first expression in Claim 3.1, we get that

$$
\begin{aligned}
m(t, \beta) D_{k} P_{2 n}(t, \beta) & =-\left(m P_{2 n}\right)(t, \beta) D_{k} \ln m(t, \beta)+\int_{\mathcal{T} \backslash[0, t)} \mathbb{E}\left[D_{k} m_{n}\left(s, \beta_{s}\right) \mid \beta_{t}=\beta\right] \mathrm{d} s \\
& =\int_{\mathcal{T} \backslash(0, t)} \mathbb{E}_{\pi}\left[D_{k} m_{n}\left(s, \beta_{s}\right)-m_{n}\left(s, \beta_{s}\right) D_{k} \ln m(t, \beta) \mid \beta_{t}=\beta\right] \mathrm{d} s \\
& =m(t, \beta) \int_{\mathcal{T} \backslash(0, t)} \mathbb{E}_{\pi}\left[m_{n}^{*}\left(\left(s, \beta_{s}\right),(t, \beta)\right) \mid \beta_{t}=\beta\right] \mathrm{d} s
\end{aligned}
$$

The remainder of the argument is the same as in the latter part of the proof for Theorem 3.2.

Regarding the cases investigated in Theorem 3.1 and Claim 3.2 above, our analysis remains valid when the price process $\left\{P_{n}\right\}_{n \in \mathcal{K}}$ derives from the risk-neutral valuation method. More precisely, when $P_{n}(\cdot)=\mathbf{1}_{\mathcal{T} \neq \mathbb{R}_{+}} \times P_{1 n}(\cdot)+P_{2 n}(\cdot)$ is given by

$$
\begin{aligned}
& P_{1 n}(t, \widetilde{\beta}):=\mathbb{E}_{\widetilde{\pi}}\left[\exp \left(-\int_{t}^{T} r_{\tau} \mathrm{d} \tau\right) G_{n}\left(T, \widetilde{\beta}_{T}\right) \mid \widetilde{\beta}_{t}=\widetilde{\beta}\right] \\
& P_{2 n}(t, \widetilde{\beta}):=\mathbb{E}_{\widetilde{\pi}}\left[\int_{\mathcal{T} \backslash(0, t)} \exp \left(-\int_{t}^{s} r_{\tau} \mathrm{d} \tau\right) g_{n}\left(s, \widetilde{\beta}_{s}\right) \mathrm{d} s \mid \widetilde{\beta}_{t}=\widetilde{\beta}\right]
\end{aligned}
$$

with $\widetilde{\beta}: \Omega \times \mathcal{T} \rightarrow \mathbb{R}^{K}$ being another $K$-dimensional Brownian motion on $(\Omega, \mathcal{F}, \tilde{\pi})$, and $\tilde{\pi}$ a martingale measure equivalent to $\pi .{ }^{9}$ Comparing these expressions with those in (2) and (5), it follows immediately that the dynamics with respect to the typical Brownian dimension are given here by (3) and the first expression in Claim 3.1 for $G_{0}(T, \cdot)=1=g_{0}(\cdot)$ and $M(t, \cdot)=\exp \left(-\int_{0}^{t} r_{s} \mathrm{~d} s\right)=m(t, \cdot)$. Theorem 3.1, Claim 3.2, and Corollary 3.1 can be stated, respectively, as follows.

Corollary 3.2 Let $\mathcal{T}=[0, T]$ for some $T \in \mathbb{R}_{++}$and suppose that the price process is given by (9) with $A 1(i)$ satisfied for $M(T, \cdot):=\exp \left(-\int_{0}^{T} r_{s} d s\right)$. Then $\left[D_{k} P_{1 n}(\cdot)\right]_{(n, k) \in \mathcal{K} \times \mathcal{K}}$ has full rank almost everywhere on $(0, T) \times \mathbb{R}^{K}$ if $\left[D_{k} G_{n}(T, \cdot)\right]_{(n, k) \in \mathcal{K} \times \mathcal{K}}$ is non-singular at some $\beta_{T} \in \mathbb{R}^{K}$.

Corollary 3.3 Let $\mathcal{T}=[0, T]$ for some $T \in \mathbb{R}_{++}$. Suppose also that the price process $P_{n}(\cdot)=$ $P_{1 n}(\cdot)+P_{2 n}(\cdot)$ is given by (9)-(10) with Al(i) satisfied for $M(T, \cdot):=\exp \left(-\int_{0}^{T} r_{s} d s\right)$.

9 The risk-neutral valuation method derives from strengthening the no-arbitrage condition-see, for instance, Proposition 6.K and Section 6.L in Duffie [11] but also Theorem 5.7.1 and the subsequent discussion in Bingham and Kiesel [6]. 
Suppose also that, for each $j \in \mathcal{K}$, a European call maturing at $T$ (with strike price some $\left.X_{j}>0\right)$ along with its equivalent put are available for trading. Then $\left[D_{k} P_{n}(\cdot)\right]_{(n, k) \in \mathcal{K} \times \mathcal{K}}$ has full rank almost everywhere on $[0, T) \times \mathbb{R}^{K}$ if $\left[D_{k} G_{n}(T, \cdot)\right]_{(n, k) \in \mathcal{K} \times \mathcal{K}}$ is non-singular at some $\beta \in \mathbb{R}^{K}$.

Corollary 3.4 Let the price process be given by (10) with Al(ii) and A2 satisfied for $m(t, \cdot):=$ $\exp \left(-\int_{0}^{t} r_{s} d s\right)$, respectively, when $\mathcal{T}=[0, T]$ for some $T \in \mathbb{R}_{++}$and $\mathcal{T}=\mathbb{R}_{++}$. Then $\left[D_{k} P_{2 n}(\cdot)\right]_{(n, k) \in \mathcal{K} \times \mathcal{K}}$ has full rank everywhere on int $(\mathcal{T}) \times \mathbb{R}^{K}$ if the collection $\left\{g_{n}(\cdot)\right\}_{n \in \mathcal{K}}$ satisfies strong non-singularity on $\prod_{n \in \mathcal{K}} S_{g_{n}}$.

\section{Discussion and related literature}

As pointed out already, the issue under investigation here has been analyzed also in Anderson and Raimondo [2], Riedel and Herzberg [28] (see also Riedel and Herzberg [27]) as well as Kramkov [19]. Anderson and Raimondo [2] considers the pricing process in (1) when the time-horizon is finite and the securities' dividends comprise both intermediate flows and terminal lump sums. The terminal lump-sum dividends and individual lump-sum endowments are taken to be continuous almost everywhere in $\{T\} \times \mathbb{R}^{K}$ while their counterparts in intermediate flows are assumed to be analytic on $(0, T) \times \mathbb{R}^{K}$. The agents' utilities are analytic, strictly increasing and strictly concave functions of the form $u\left(c\left(t, \beta_{t}\right), t, \beta_{t}\right)$, which satisfy in addition the Inada conditions uniformly. In Riedel and Herzberg [28], this setting is extended by allowing the underlying risk process to be a time-homogenous diffusion. Attention is restricted though to the case in which the dividends and the individual endowments are time homogenous, one of the securities is a zero-coupon bond maturing at the terminal date, the aggregate endowment is bounded, while the agents' utilities are state independent and depend on time only via an "impatience" rate. In Kramkov [19], on the other hand, the setting in Anderson and Raimondo [2] is extended to allow for general diffusions . Yet this is done at the expense of restricting the agents' relative risk aversion coefficients over terminal lump sums to be bounded while their utilities over flows and their endowment flows are time homogenous.

With respect to growth conditions, Anderson and Raimondo [2] imposes one that is stronger than that in our Assumption A1 on the dividends, the agents' marginal utilities and endowments (both in terms of flows and lump sums) as well as on the instantaneous dispersion of the dividend and endowment flows. In Riedel and Herzberg [28] these quantities are all bounded while in Kramkov [19] the same condition as in Anderson and Raimondo [2] applies on the agents' utilities, marginal utilities and endowments (both in terms of flows and lump sums) as well as on the instantaneous dispersion of the terminal lump-sum dividends.

The above features not withstanding, in all of the aforementioned studies, the sufficient condition for the respective financial market to be dynamically complete is the same as that in our Theorem 3.1. And despite the presence of intermediate dividend flows, the intuition for the sufficiency of the non-degeneracy condition in question is also the same as that in our proof for Theorem 3.1. Specifically, even though $P_{j}(\cdot)=P_{1 j}(\cdot)+P_{2 j}(\cdot)$, as there is no value left to a security on the terminal date other than its lump-sum dividend, we do have $\lim _{t \rightarrow T} P_{j}(t, \cdot)=$ $\lim _{t \rightarrow T} P_{1 j}(t, \cdot)$ and, thus, $\lim _{t \rightarrow T} J_{p}(\mathcal{I}(\omega, t))=\left[D_{k} G_{n / 0}(\mathcal{I}(\omega, T))\right]_{(n, k) \in \mathcal{K} \times \mathcal{K}}$ almost everywhere on $\Omega$. Moreover, since all intermediate dividend and marginal utility flows are analytic on $[0, T) \times \mathbb{R}^{K}$, so is the integral $P_{2 j}(\cdot)$. As a result, $P_{j}(\cdot)$ and, thus, also $\left|J_{p}(\cdot)\right|$ are analytic on $[0, T) \times \mathbb{R}^{K}$. Clearly, the latter part of our argument in the proof for Theo- 
rem 3.1 remains valid when $P_{1 j}(\cdot)$ [resp. $p_{1 j}(\cdot)$ ] is replaced by $P_{j}(\cdot)$ [resp. $p_{j}(\cdot)$ ] under analyticity. ${ }^{10}$

This line of reasoning depicts also the intuition behind the non-degeneracy condition in Hugonnier et al. [17]. Compared to the aforementioned papers, this study extends the setting in Riedel and Herzberg [27] by allowing the underlying risk process to follow a general diffusion and the time horizon to be infinite (albeit, in the latter case, under the restriction that the agents' relative risk aversion coefficients are bounded). The main analysis in Hugonnier et al. [17] is conducted under a finite time horizon in the presence of a money-market account and only dividend flows. The sufficient condition for the financial market to be dynamically complete applies on the dispersion matrix of the flow-dividends: $\left[D_{k} g_{n}(\cdot)\right]_{(n, k) \in \mathcal{K} \times \mathcal{K}}$ is taken to be non-degenerate at some $(t, \beta) \in(0, T) \times \mathbb{R}^{K}$ with $t$ being arbitrarily close to $T$. The underlying intuition emerges now in light of our expessions in Claim 3.1.

Recall in particular the first expression in Claim 3.1. Letting $t$ be arbitrarily close to $T$, the dispersion of the typical security price can be approximated by the relation

$$
\begin{aligned}
m\left(t, \beta_{t}\right) D_{k} P_{2 n}\left(t, \beta_{t}\right) & =-P_{2 n}\left(t, \beta_{t}\right) D_{k} m\left(t, \beta_{t}\right)-D_{k} m_{n}\left(t, \beta_{t}\right)(T-t) \\
& =-\left(P_{n}\left(t, \beta_{t}\right)+g_{n}\left(t, \beta_{t}\right)(T-t)\right) D_{k} m\left(t, \beta_{t}\right)-m\left(t, \beta_{t}\right) D_{k} g_{n}\left(t, \beta_{t}\right) \\
& =-m\left(t, \beta_{t}\right) D_{k} g_{n}\left(t, \beta_{t}\right)(T-t)
\end{aligned}
$$

where the first and last equalities above follow by the fact that the time integrals vanish as we approach the terminal date: we have $\left(m P_{2 n}\right)(t, \beta)=-\mathbb{E}_{\pi}\left[m_{n}\left(t, \beta_{t}\right) \mid \beta_{t}=\beta\right](T-t)+$ $o(T-t)=-m_{n}(t, \beta)(T-t)+o(T-t)$, and thus also $P_{2 n}(t, \beta)=-g_{n}(t, \beta)(T-t)+$ $o(T-t)$. Clearly, if $\left[D_{k} g_{n}(t, \beta)\right]_{(n, k) \in \mathcal{K} \times \mathcal{K}}$ is non-singular then so will be $J_{P_{2}}(t, \beta)$ - the remainder of the argument being the same as above but for replacing $P_{n}(\cdot)$ with $P_{2 n}(\cdot) .^{11}$

It is noteworthy of course that, even though our Corollary 3.1 refers to a financial setting that is consistent with that underpinning the main analysis in Hugonnier et al. [17], the respective non-degeneracy conditions coincide only locally. To see this, observe that $\left[D_{k} g_{n}(t, \beta)\right]_{(n, k) \in \mathcal{K} \times \mathcal{K}}$ is non-singular only if the matrix

$$
\left[m_{1}^{*}((t, \beta),(t, \beta)) \cdots m_{K}^{*}((t, \beta),(t, \beta))\right]
$$

is also non-singular. Yet the latter property necessitates in turn the existence of some neighbourhood $V$ of $(t, \beta)$ in $(0, T) \times \mathbb{R}^{K}$ such that

$$
\left[m_{1}^{*}\left(\left(s_{1}, \beta_{s_{1}}\right),\left(\tau_{1}, \beta_{\tau_{1}}\right)\right) \cdots m_{K}^{*}\left(\left(s_{K}, \beta_{s_{K}}\right),\left(\tau_{K}, \beta_{\tau_{K}}\right)\right)\right]
$$

remains non-singular for any collection $\left\{\left(s_{k}, \beta_{s_{k}}\right),\left(\tau_{k}, \beta_{\tau_{k}}\right)\right\}_{k \in \mathcal{K}}$ from $V$ (see Claim C.1 in the "Appendix"). Hence, the requirement that $\left[D_{k} g_{n}(t, \beta)\right]_{(n, k) \in \mathcal{K} \times \mathcal{K}}$ is non-singular guarantees in fact that $\left\{m_{n}^{*}\right\}_{k \in \mathcal{K}}$ satisfies strong non-singularity on $V$; equivalently, that $\left[D_{k} P_{2 n}(\cdot)\right]_{(n, k) \in \mathcal{K} \times \mathcal{K}}$ has full rank everywhere on $V$ (recall our Corollary 3.1). Similarly, even though Claim 3.2 refers to a financial setting that is consistent with those in Anderson and Raimondo [2], Riedel and Herzberg [28] or Kramkov [19], we had to assume the availability of options in order to establish the same sufficiency result. Either of these observations attests to the mileage one gets out of analyticity. Equally importantly perhaps, the obvious

\footnotetext{
$\overline{10}$ For a more detailed description of the argument outlined above, see Appendices B and D in Anderson and Raimondo [2].

11 The same argument applies also in the absence of a money-market account. The second expression in Claim 3.1 leads to the approximation: $m(t, \beta)^{2} D_{k} p_{n}(t, \beta)=-m(t, \beta)^{2} D_{k} g_{n / 0}(t, \beta)(T-\tau)^{2}$. In this case, it suffices that $\left[D_{k} g_{n / 0}(t, \beta)\right]_{(n, k) \in \mathcal{K} \times \mathcal{K}}$ is non-singular.
} 
reason why our argument in the proof of Theorem 3.1 cannot apply also in the settings of Theorem 3.2 or Claim 3.2 offers an intuitive understanding of the claim that one may do away with assuming analyticity in the space variables, yet analyticity in time remains sine qua non. ${ }^{12}$

Yet another insight born out of the relation our analysis bears to the aforementioned papers is the indication that the sufficiency of the respective non-degeneracy conditions extends beyond pure-exchange economies. In the context of general equilibrium analysis, the pricing kernel cannot be but a weighted average of the agents' equilibrium marginal utilities. This notwithstanding, the asset-pricing equation in (1) allows also for non-financial wealth (and, thus, production). In this sense, the essential premise is that the time- and state-dependency of the primitive variables (utilities, dividends, endowments, and other non-financial wealth) obtains as a function of $\left(t, \beta_{t}\right)$. As an approach towards equilibrium asset-pricing theory this has been the building block for much of the seminal literature.

The starting point was to assume that the agents have identical preferences. This has been the launching pad of two related strands of the literature. The first restricts attention to what is essentially the continuous-time analogue of the static (one-period) model: the time horizon is finite and securities pay only lump-sum dividends on the terminal date. ${ }^{13}$ The second approach has been to allow for securities that pay also dividend flows during the time interval while the time horizon may be infinite. ${ }^{14}$ The next step in the literature was to study agents with heterogenous preferences. Even in this case, however, the pricing kernel remains a linear function of the equilibrium marginal utilities (the Negishi weights are constant) if the equilibrium allocation is Pareto-optimal. ${ }^{15}$ As a result, even in this case the pricing formula retains the same basic form as that under the present investigation. ${ }^{16}$

Clearly, in the context of general equilibrium, as long as we maintain Pareto optimality as a desideratum, the scope of our analysis is large. By considering general formulations for the pricing kernel and the securities' dividends, our analysis applies irrespectively of preferences, endowments, and other structural elements (such as whether the agents' budget constraints include only pure exchange). And this is important in its own right; it has become by now clear in the literature that the choice of pricing kernel can play a crucial role for the model's results. ${ }^{17}$

Equally importantly, our analysis remains valid even in the presence of multiple underlying consumption commodities. With more than one consumption commodities, the dividend specifications in (1) would be given as $\widetilde{G}_{j}(\cdot)=\left(Q_{j} G_{j}\right)(\cdot)$ and $\widetilde{g}_{j}(\cdot)=\left(q_{j} g_{j}\right)(\cdot)$ with

12 For enlightening discussions on this point, see Section A in the Supplementary Appendix to Hugonnier et al. [17] or Remark 3.4 in Kramkov [19].

13 Our pricing Eq. (2) can be found, for instance, in Bick [4,5], He and Leland [16], Raimondo [25] or Anderson and Raimondo [1].

14 Perhaps the most well-known paper in this strand is Cox et al. [8], the continuous-time analogue of the famous model in Lucas [21] enhanced to include production. Our pricing Eq. (5) is consistent with the analysis in Cox et al. [8]; it can be found also in Cochrane et al. [7], Martin [22], Farhi and Panageas [15], Merton [23] (see Chapters 4-5) or Wang [30].

15 When the equilibrium allocation is not Pareto-optimal, the representative agent's utility function will be state-dependent even if all individuals have state-independent preferences and homogenous beliefs (see, for example, Cuoco and He [9]). In fact, the Negishi weights in the construction of the representative agent may even play the role of endogenous state variables which cannot be recovered as functions of the exogenous ones.

16 Our pricing formula (1) can be found in Basak and Cuoco [3], Duffie and Zaime [12] (see Theorem 1 and the subsequent discussion in Sect. 5), Dumas [13], Karatzas et al. [18] (see Corollary 10.4) or Riedel [26] (Theorem 2.1).

17 See footnote 2 in Riedel and Herzberg [28] for relevant references. 
$Q_{j}(\cdot)$ and $q_{j}(\cdot)$ being, respectively, the terminal and intermediate values for the relative price (with respect to that of the numeraire commodity) of the commodity in whose units the dividend of the $j$ th security is paid. ${ }^{18}$ Obviously, our results remain valid under these dividend specifications. Yet the latter entail also endogenously determined quantities; the commodity prices $Q_{j}(\cdot)$ and $q_{j}(\cdot)$ are determined endogenously by the equilibrium itself. As a result, apart from very special cases, the specifications of the securities' dividends become conditional on the choice of numeraire. Needless to say, the latter qualification applies also for the sufficiency of our non-degeneracy conditions; they guarantee that the financial market will be dynamically complete as long as the normalization remains with respect to the given numeraire.

\section{Concluding remarks}

In an Arrow-Debreu economy, the agents may shift consumption or income across states and time by trading a complete set of contingent claims, once and for all at the beginning of time. When they are instead constrained to trade a given set of securities, the market is said to be dynamically complete if repeated trading of the securities can still deliver any allocation that would be feasible under a complete set of contingent claims. Under continuous-time trading, this may be possible by trading a finite set of securities rapidly enough, even though the information about the state of the world is revealed through a stochastic process. In particular, when the underlying uncertainty is driven by Brownian motions, this can happen if the securities' market is potentially dynamically complete (i.e., the number of securities exceeds that of independent Brownian motions by at least one). ${ }^{19}$

However, potential dynamic completeness does not suffice by itself: some form of independence amongst the securities' payoffs must obtain in addition. In general, once the securities' prices are appropriately deflated, this refers to the non-degeneracy of their instantaneous dispersion with respect to the underlying stochastic process. The present paper establishes sufficient conditions for such non-degeneracy when the pricing process is given by (1) - as long as some standard in the literature growth conditions obtain. ${ }^{20}$

Of course, our attention was restricted to the exogenous risk process being a Brownian motion while the time- and state-dependency of the primitive variables obtain as functions of $\left(t, \beta_{t}\right)$. And even though this combination has been used extensively in the literature, it does mean that our results do not extend to a larger class of stochastic models (such as OrnsteinUhlenbeck or more generally affine processes) that is becoming increasingly the forefront of the research in financial economics. Nevertheless, the setting of the present study has always been an important theoretical benchmark in the quest for fundamental equilibrium insight. The significance of being able to determine explicitly if and when dynamic completeness obtains in this setting is obvious.

Open Access This article is licensed under a Creative Commons Attribution 4.0 International License, which permits use, sharing, adaptation, distribution and reproduction in any medium or format, as long as you give appropriate credit to the original author(s) and the source, provide a link to the Creative Commons licence, and indicate if changes were made. The images or other third party material in this article are included in the

\footnotetext{
18 See, for instance, the formulations in Ehling and Heyerdahl-Larsen [14].

19 When the underlying stochastic process is not Brownian, the required number of securities may be larger.

20 The growth condition in Assumptions A1-A2 is used for the study of the heat equation-see, for example, Appendix C in Nielsen [24].
} 
article's Creative Commons licence, unless indicated otherwise in a credit line to the material. If material is not included in the article's Creative Commons licence and your intended use is not permitted by statutory regulation or exceeds the permitted use, you will need to obtain permission directly from the copyright holder. To view a copy of this licence, visit http://creativecommons.org/licenses/by/4.0/.

\section{Appendices}

\section{A Supporting results}

Taking $(\delta, \mathbf{x}) \in \mathbb{R}_{++} \times \mathbb{R}^{K}, \mathcal{B}_{\mathbf{x}}(\delta)$ denotes in what follows the open ball in $\mathbb{R}^{K}$ of radius $\delta$ that is centered at $\mathbf{x}$.

Lemma A.1 Let the functions $\lambda: \mathbb{R}^{K} \rightarrow(0,1]$ and $f: \mathbb{R}^{K} \rightarrow \mathbb{R}$ be continuous. Suppose also that

$$
\exists \delta_{0}, r_{0} \in \mathbb{R}_{++}:|f(\mathbf{x})| \leq r_{0}+e^{r_{0}|\mathbf{x}|} \quad \forall \mathbf{x} \in \mathbb{R}^{K} \backslash \mathcal{B}_{\mathbf{0}}\left(\delta_{0}\right)
$$

Then

$$
\forall r \in \mathbb{R}_{++}, \exists C \in \mathbb{R}_{++}:|f(\lambda(\mathbf{x}) \mathbf{x})|<C e^{r|\mathbf{x}|^{2}} \quad \forall \mathbf{x} \in \mathbb{R}^{K}
$$

Proof Take an arbitrary $r \in \mathbb{R}_{++}$. By hypothesis, there exist $r_{0}, \delta_{0}>0$ such that

$$
|f(\lambda(\mathbf{x}) \mathbf{x})| \leq r_{0}+e^{r_{0}|\lambda(\mathbf{x}) \mathbf{x}|} \leq r_{0}+e^{r_{0}|\mathbf{x}|}
$$

for any $\mathbf{x} \in \mathbb{R}^{K}:|\mathbf{x}| \geq \delta_{0}$. For all $\mathbf{x} \in \mathbb{R}^{K}:|\mathbf{x}| \geq r_{0} / r$, however, we have in addition that

$$
r_{0}+e^{r_{0}|\mathbf{x}|} \leq r_{0}+e^{r|\mathbf{x}|^{2}} \leq\left(1+r_{0}\right) e^{r|\mathbf{x}|^{2}}
$$

Letting, therefore, $\delta=\max \left\{\delta_{0}, r_{0} / r\right\}$ we have that

$$
|f(\lambda(\mathbf{x}) \mathbf{x})|<\left(1+r_{0}\right) e^{r|\mathbf{x}|^{2}} \quad \forall \mathbf{x} \in \mathbb{R}^{K} \backslash \mathcal{B}_{\mathbf{0}}(\delta)
$$

Yet, $\lambda(\cdot)$ and $f(\cdot)$ being both continuous, there exists $C_{0}>0$ such that $|f(\lambda(\mathbf{x}) \mathbf{x})| \leq C_{0} \leq$ $C_{0} e^{r|\mathbf{x}|^{2}}$ for any $\mathbf{x} \in \mathcal{B}_{\mathbf{0}}(\delta)$. The claim follows by setting $C=\max \left\{C_{0}, 1+r_{0}\right\}$.

Lemma A.2 Let $(\tau, \beta) \in \mathbb{R}_{++} \times \mathbb{R}^{K}$ be a parameter vector. Suppose also that $f: \mathbb{R}^{K} \rightarrow \mathbb{R}$ satisfies

$$
|f(\mathbf{x})| \leq C_{0} e^{r_{0}|\mathbf{x}|^{2}} \quad \forall \mathbf{x} \in \mathbb{R}^{K}
$$

for some constants $\left(r_{0}, C_{0}\right) \in(0, \tau / 2) \times \mathbb{R}_{++}$. Then there exist constants $r, \delta \in \mathbb{R}_{++}$such that

$$
|f(\mathbf{x}) \phi(\sqrt{\tau}(\mathbf{x}-\beta))| \leq C_{0} \phi(\sqrt{2 r} \mathbf{x}) \quad \forall \mathbf{x} \in \mathbb{R}^{K} \backslash \mathcal{B}_{\mathbf{0}}(\delta)
$$

Proof Observe first that, given any $\alpha>0$, we have

$$
\left|\mathbf{x}^{\top} \beta\right| \leq|\mathbf{x}| \times|\beta| \leq \alpha|\mathbf{x}|^{2} \quad \forall \mathbf{x} \in \mathbb{R}^{K}:|\mathbf{x}| \geq|\beta| / \alpha
$$

This implies in turn that

$$
\begin{aligned}
r_{0}|\mathbf{x}|^{2}-\frac{\tau}{2}|\mathbf{x}-\beta|^{2} & =\left(r_{0}-\frac{\tau}{2}\right)|\mathbf{x}|^{2}-\frac{\tau}{2}|\beta|^{2}+\tau \mathbf{x}^{\top} \beta \\
& \leq\left(r_{0}-\frac{\tau}{2}\right)|\mathbf{x}|^{2}+\tau \mathbf{x}^{\top} \beta \\
& \leq\left(r_{0}+\tau\left(\alpha-\frac{1}{2}\right)\right)|\mathbf{x}|^{2} \quad \forall \mathbf{x} \in \mathbb{R}^{K}:|\mathbf{x}| \geq|\beta| / \alpha
\end{aligned}
$$


Choosing, therefore, $\alpha \in\left(0, \frac{\tau-2 r_{0}}{2 \tau}\right)$ establishes that

$$
|f(\mathbf{x}) \phi(\sqrt{\tau}(\mathbf{x}-\beta))|=\frac{1}{\sqrt{(2 \pi)^{K}}}|f(\mathbf{x})| e^{-\frac{\tau}{2}|\mathbf{x}-\beta|^{2}} \leq \frac{C_{0}}{\sqrt{(2 \pi)^{K}}} e^{-r|\mathbf{x}|^{2}} \quad \forall \mathbf{x} \in \mathbb{R}^{K} \backslash \mathcal{B}_{\mathbf{0}}(|\beta| / \alpha)
$$

where $r:=\left(\frac{1}{2}-\alpha\right) \tau-r_{0}>0$. To complete the argument let $\delta:=|\beta| / \alpha$.

Lemma A.3 Given a measure space $(X, \mathcal{A}, \mu)$ and a non-empty, non-degenerate open interval $(a, b) \subseteq \mathbb{R}$, let $f:(a, b) \times X \rightarrow \mathbb{R}$ be a function satisfying ${ }^{21}$

(i) $x \rightarrow f(t, x)$ is $\mu$-integrable on $X$ for every fixed $t \in(a, b)$,

(ii) $t \rightarrow f(t, x)$ is continuous for every fixed $x \in X$, and

(iii) there exists a function $h: X \rightarrow \mathbb{R}_{+}$such that $\int_{X} h(x) d \mu(x)<+\infty$ and $|f(t, x)|<$ $h(x)$ for all $(t, x) \in(a, b) \times X$.

Then the function $v:(a, b) \rightarrow \mathbb{R}$ given by

$$
t \rightarrow v(t):=\int_{X} f(t, x) d \mu(x)
$$

is continuous.

Lemma A.4 Let $(\tau, \beta) \in \mathbb{R}_{++} \times \mathbb{R}^{K}$ be a parameter vector. Suppose also that the continuous function $f: \mathbb{R}^{K} \rightarrow \mathbb{R}$ satisfies

$$
|f(\mathbf{x})| \leq C e^{r|\mathbf{x}|^{2}} \quad \forall \mathbf{x} \in \mathbb{R}^{K}
$$

for some constants $(r, C) \in(0, \tau / 2) \times \mathbb{R}_{++}$. Letting then $\mathbf{m}=\left(m_{1}, \ldots, m_{K}\right) \in \mathbb{N}^{K}$, the $\mathbb{R}^{K} \rightarrow \mathbb{R}$ function

$$
F^{(\mathbf{m})}(\beta)=\int_{\mathbb{R}^{K}} f(\mathbf{x}) \frac{\partial^{\sum_{k \in \mathcal{K}} m_{k}} \phi(\sqrt{\tau}(\mathbf{x}-\beta))}{\prod_{k \in \mathcal{K}} \partial \beta_{k}^{m_{k}}} d \mathbf{x}
$$

is well defined and $C^{1}$, with its partial derivatives given by

$$
\frac{\partial F^{(\mathbf{m})}(\beta)}{\partial \beta_{k}}=\int_{\mathbb{R}^{K}} f(\mathbf{x}) \frac{\partial^{1+\sum_{k \in \mathcal{K}} m_{k}} \phi(\sqrt{\tau}(\mathbf{x}-\beta))}{\partial \beta_{k}^{m_{k}+1} \prod_{l \in \mathcal{K} \backslash\{k\}} \partial \beta_{l}^{m_{l}}} d \mathbf{x} \quad k \in \mathcal{K}
$$

Proof Fix an arbitrary $\beta \in \mathbb{R}^{K}$ and an arbitrary dimension $k \in \mathcal{K}$. Our argument-which will focus upon the interval $\left(\beta_{k}-\epsilon_{k}, \beta_{k}+\epsilon_{k}\right)$ for some $\epsilon_{k} \in(0,1)$-will be presented in steps.

Step 1(i). If $\mathbf{m}=\mathbf{0}^{K}$, that $F^{(\mathbf{m})}(\cdot)$ is well defined follows immediately from Lemma A.2 above. $^{22}$ For the case $\mathbf{m} \neq \mathbf{0}^{K}$, notice first that a well-known property of the pdf of a scalar standard normal variable is that its $n$th derivative is given by

$$
\mathrm{d}^{n} \phi\left(x_{k}\right) / \mathrm{d} x_{k}^{n}=H_{n}\left(x_{k}\right) \phi\left(x_{k}\right), \quad x_{k} \in \mathbb{R}, n \in \mathbb{N}
$$

\footnotetext{
21 This result is well-known in the literature as the continuity lemma for parameter-dependent integrals. The present statement appears, for instance, as Theorem 11.4 in Schilling [29].
}

22 Recall that the zeroth derivative of a function denotes the function itself. 
with $H_{n}(\cdot)$ being the Hermite polynomial of order $n$. It follows therefore that, writing $H_{n}\left(x_{k}\right)=\sum_{i=0}^{n} c_{i} x_{k}^{i}$ for some constants $c_{0}, \ldots, c_{n} \in \mathbb{R}$, we have

$$
\begin{aligned}
\left|\mathrm{d}^{n} \phi\left(x_{k}\right) / \mathrm{d} x_{k}^{n}\right|=\left|H_{n}\left(x_{k}\right)\right| \phi\left(x_{k}\right) & \leq \phi\left(x_{k}\right) \sum_{i=0}^{n}\left|c_{i}\right||x|_{k}^{i} \\
& \leq C_{n}\left|x_{k}\right|^{n} \phi\left(x_{k}\right) \\
& \leq C_{n} \phi\left(x_{k}\right) e^{n\left|x_{k}\right|} \leq C_{n} \phi\left(x_{k}\right) e^{\alpha x_{k}^{2} / 2}=C_{n} \phi\left((1-\alpha) x_{k}\right)
\end{aligned}
$$

for any $\alpha>0$, all $x_{k} \in \mathbb{R} \backslash(-1,1):\left|x_{k}\right| \geq 2 n / \alpha$ and where $C_{n}=\max \left\{\left|c_{i}\right|: i \in\{0, \ldots, n\}\right\}$. It is trivial to check moreover that, letting $g_{n}\left(\sqrt{\tau} x_{k}\right):=\sqrt{\tau^{n}}\left(H_{n} \phi\right)\left(\sqrt{\tau} x_{k}\right)$ for $\left(n, x_{k}\right) \in$ $\mathbb{N} \times \mathbb{R}$, we have

$$
\begin{aligned}
\mathrm{d}^{n} \phi\left(\sqrt{\tau}\left(x_{k}-\beta_{k}\right)\right) / \mathrm{d} x_{k}^{n} & =\sqrt{\tau^{n}} H_{n}\left(\sqrt{\tau}\left(x_{k}-\beta_{k}\right)\right) \phi\left(\sqrt{\tau}\left(x_{k}-\beta_{k}\right)\right) \\
& =g_{n}\left(\sqrt{\tau}\left(x_{k}-\beta_{k}\right)\right), \quad\left(n, x_{k}\right) \in \mathbb{N} \times \mathbb{R}
\end{aligned}
$$

And regarding the domain-restrictions above, we have that

$$
\begin{aligned}
\sqrt{\tau}\left|x_{k}-\beta_{k}-\widetilde{z}_{k}\right| \geq \sqrt{\tau}\left(\left|x_{k}\right|-\left|\beta_{k}\right|-\left|\widetilde{z}_{k}\right|\right) & >\sqrt{\tau}\left(\left|x_{k}\right|-\left|\beta_{k}\right|-\epsilon_{k}\right) \\
& \geq \sqrt{\tau}\left(\left|x_{k}\right|-\left|\beta_{k}\right|-1\right) \geq 2 n / \alpha
\end{aligned}
$$

for any $\widetilde{z}_{k} \in\left(-\epsilon_{k}, \epsilon_{k}\right)$ and any $x_{k} \in \mathbb{R}:\left|x_{k}\right| \geq 1+\frac{2 n}{\alpha \sqrt{\tau}}+\left|\beta_{k}\right|$.

Step 1(ii). It follows from the preceding step that

$$
\frac{\partial^{\sum_{k=1}^{K} m_{k}} \phi(\sqrt{\tau}(\mathbf{x}-\beta))}{\prod_{k=1}^{K} \partial \beta_{k}^{m_{k}}}=\prod_{k=1}^{K} g_{m_{k}}\left(\sqrt{\tau}\left(x_{k}-\beta_{k}\right)\right)
$$

for all $\mathbf{x} \in \mathbb{R}^{K}$ and all $\mathbf{m} \in \mathbb{N}^{K}$, while

$$
\left|g_{m_{k}}\left(\sqrt{\tau}\left(x_{k}-\beta_{k}-\widetilde{z}_{k}\right)\right)\right| \leq C_{m_{k}} \phi\left(\sqrt{\tau(1-\alpha)}\left(x_{k}-\beta_{k}-\widetilde{z}_{k}\right)\right)
$$

for any $\alpha>0$, any $\widetilde{z}_{k} \in\left(-\epsilon_{k}, \epsilon_{k}\right)$, and any $x_{k} \in \mathbb{R} \backslash\left(-\delta_{k}, \delta_{k}\right)$ where $\delta_{k}=1+\frac{2 m_{k}}{\alpha \sqrt{\tau}}+\left|\beta_{k}\right|$. Set now $\widetilde{z}_{k}=0$ for all $k \in \mathcal{K}$, and let $m_{0}=\max \left\{m_{k}: k \in \mathcal{K}\right\}$ and $\beta_{0}=\max \left\{\left|\beta_{k}\right|: k \in \mathcal{K}\right\}$. Then

$$
\begin{aligned}
\left|\frac{\partial^{\sum_{k=1}^{K} m_{k}} \phi(\sqrt{\tau}(\mathbf{x}-\beta))}{\prod_{k=1}^{K} \partial \beta_{k}^{m_{k}}}\right| & \leq \prod_{k=1}^{K} \sqrt{\tau^{m_{k}}} C_{m_{k}} \phi\left(\sqrt{\tau(1-\alpha)}\left(x_{k}-\beta_{k}\right)\right) \\
& =C_{0}^{\prime} \phi(\sqrt{\tau(1-\alpha)}(\mathbf{x}-\beta)) \quad \forall \mathbf{x} \in \mathbb{R}^{K} \backslash \mathcal{B}_{\mathbf{0}}\left(\delta_{0}\right)
\end{aligned}
$$

where $\delta_{0}=1+\frac{2 m_{0}}{\alpha \sqrt{\tau}}+\beta_{0}$ and $C_{0}^{\prime}=\prod_{k=1}^{K} \sqrt{\tau^{m_{k}}} C_{m_{k}}$.

Step 1(iii). We are now in position to show that $F^{(\mathbf{m})}(\cdot)$ is well defined. Taking $\alpha \in(0,1)$, (14) above implies that

$$
\left|f(\mathbf{x}) \frac{\partial^{\sum_{k=1}^{K} m_{k}} \phi(\sqrt{\tau}(\mathbf{x}-\beta))}{\prod_{k=1}^{K} \partial \beta_{k}^{m_{k}}}\right| \leq C_{0}^{\prime}|f(\mathbf{x})| \phi(\sqrt{\tau(1-\alpha)}(\mathbf{x}-\beta)) \quad \forall \mathbf{x} \in \mathbb{R}^{K} \backslash \mathcal{B}_{\mathbf{0}}\left(\delta_{0}\right)
$$

By hypothesis, moreover, there are $r \in(0, \tau / 2)$ and $C>0$ such that $|f(\mathbf{x})| \leq C e^{r|\mathbf{x}|^{2}}$ on $\mathbb{R}^{K}$. Choosing, therefore, $\alpha \in\left(0, \frac{\tau-2 r}{\tau}\right)$ so that $r<\frac{\tau(1-\alpha)}{2}$, Lemma A.2 above ensures the 
existence of $r^{\prime}, \delta_{0}^{\prime}>0$ such that

$$
\left|f(\mathbf{x}) \frac{\partial^{\sum_{k=1}^{K} m_{k}} \phi(\sqrt{\tau}(\mathbf{x}-\beta))}{\prod_{k=1}^{K} \partial \beta_{k}^{m_{k}}}\right| \leq C C_{0}^{\prime} \phi\left(\sqrt{r^{\prime}} \mathbf{x}\right) \quad \forall \mathbf{x} \in \mathbb{R}^{K} \backslash \mathcal{B}_{\mathbf{0}}\left(\max \left\{\delta_{0}, \delta_{0}^{\prime}\right\}\right)
$$

And as the functions $f, \phi$, and $H_{m_{k}}$ are all continuous with respect to $\mathbf{x}$, the claim follows. Step 2(i). We will show next that the partial derivatives exist and are given by the expression in the statement. Observe that, by (12) above, we have

$$
\begin{aligned}
& \left|\frac{F^{(\mathbf{m})}\left(\beta_{k}+z_{k}, \beta_{-k}\right)-F^{(\mathbf{m})}(\beta)}{z_{k}}-\int_{\mathbb{R}^{K}} f(\mathbf{x}) \frac{\partial^{m_{k}+1+\sum_{l \in \mathcal{K} \backslash\{k\}} m_{l}} \phi(\sqrt{\tau}(\mathbf{x}-\beta))}{\partial \beta_{k}^{m_{k}+1} \prod_{l \in \mathcal{K} \backslash\{k\}} \partial \beta_{l}^{m_{l}}} \mathrm{~d} \mathbf{x}\right| \\
& =\left|\int_{\mathbb{R}^{K}} f(\mathbf{x})\left[\begin{array}{l}
\prod_{l \in \mathcal{K} \backslash\{k\}} g_{m_{l}}\left(\sqrt{\tau}\left(x_{l}-\beta_{l}\right)\right) \\
\times\left(\frac{g_{m_{k}}\left(\sqrt{\tau}\left(x_{k}-\beta_{k}-z_{k}\right)\right)-g_{m_{k}}\left(\sqrt{\tau}\left(x_{k}-\beta_{k}\right)\right)}{z_{k}}-\frac{\partial g_{m_{k}}\left(\sqrt{\tau}\left(x_{k}-\beta_{k}\right)\right)}{\partial \beta_{k}}\right)
\end{array}\right] \mathrm{d} \mathbf{x}\right| \\
& \leq \int_{\mathbb{R}^{K}}\left|\begin{array}{l}
f(\mathbf{x}) \prod_{l \in \mathcal{K} \backslash\{k\}} g_{m_{l}}\left(\sqrt{\tau}\left(x_{l}-\beta_{l}\right)\right) \\
\times\left(\frac{g_{m_{k}}\left(\sqrt{\tau}\left(x_{k}-\beta_{k}-z_{k}\right)\right)-g_{m_{k}}\left(\sqrt{\tau}\left(x_{k}-\beta_{k}\right)\right)}{z_{k}}-\frac{\partial g_{m_{k}}\left(\sqrt{\tau}\left(x_{k}-\beta_{k}\right)\right)}{\partial \beta_{k}}\right)
\end{array}\right| \mathrm{d} \mathbf{x}
\end{aligned}
$$

By the mean value theorem, however, there exist $\gamma, \rho \in(0,1)$ such that

$$
\begin{aligned}
& \int_{\mathbb{R}^{K}} \begin{array}{l}
f(\mathbf{x}) \prod_{l \in \mathcal{K} \backslash\{k\}} g_{m_{l}}\left(\sqrt{\tau}\left(x_{l}-\beta_{l}\right)\right) \\
\times\left(\frac{g_{m_{k}}\left(\sqrt{\tau}\left(x_{k}-\beta_{k}-z_{k}\right)\right)-g_{m_{k}}\left(\sqrt{\tau}\left(x_{k}-\beta_{k}\right)\right)}{z_{k}}-\frac{\partial g_{m_{k}}\left(\sqrt{\tau}\left(x_{k}-\beta_{k}\right)\right)}{\partial \beta_{k}}\right)
\end{array} \mid \mathrm{d} \mathbf{x} \\
& =\int_{\mathbb{R}^{K}}\left|f(\mathbf{x}) \prod_{l \in \mathcal{K} \backslash\{k\}} g_{m_{l}}\left(\sqrt{\tau}\left(x_{l}-\beta_{l}\right)\right)\right|\left|\frac{\partial g_{m_{k}}\left(\sqrt{\tau}\left(x_{k}-\beta_{k}-\gamma z_{k}\right)\right)}{\partial \beta_{k}}-\frac{\partial g_{m_{k}}\left(\sqrt{\tau}\left(x_{k}-\beta_{k}\right)\right)}{\partial \beta_{k}}\right| \mathrm{d} \mathbf{x} \\
& =\left|\gamma z_{k}\right| \int_{\mathbb{R}^{K}}\left|f(\mathbf{x}) \prod_{l \in \mathcal{K} \backslash\{k\}} g_{m_{l}}\left(\sqrt{\tau}\left(x_{l}-\beta_{l}\right)\right) \frac{\partial^{2} g_{m_{k}}\left(\sqrt{\tau}\left(x_{k}-\beta_{k}-\gamma \rho z_{k}\right)\right)}{\partial \beta_{k}^{2}}\right| \mathrm{d} \mathbf{x} \\
& <\left|z_{k}\right| \int_{\mathbb{R}^{K}}\left|f(\mathbf{x}) \prod_{l \in \mathcal{K} \backslash\{k\}} g_{m_{l}}\left(\sqrt{\tau}\left(x_{l}-\beta_{l}\right)\right) \frac{\partial^{2} g_{m_{k}}\left(\sqrt{\tau}\left(x_{k}-\beta_{k}-\gamma \rho z_{k}\right)\right)}{\partial \beta_{k}^{2}}\right| \mathrm{d} \mathbf{x} \\
& =\left|z_{k}\right| \int_{\mathbb{R}^{K}}\left|f(\mathbf{x}) \prod_{l \in \mathcal{K} \backslash\{k\}} g_{m_{l}}\left(\sqrt{\tau}\left(x_{l}-\beta_{l}\right)\right) g_{m_{k}+2}\left(\sqrt{\tau}\left(x_{k}-\beta_{k}-\gamma \rho z_{k}\right)\right)\right| \mathrm{d} \mathbf{x}
\end{aligned}
$$

Taking thus $z_{k} \in\left(-\epsilon_{k}, \epsilon_{k}\right) \backslash\{0\}$, we have

$$
\begin{aligned}
& \left|\frac{F^{(\mathbf{m})}\left(\beta_{k}+z_{k}, \beta_{-k}\right)-F^{(\mathbf{m})}(\beta)}{z}-\int_{\mathbb{R}^{K}} f(\mathbf{x}) \frac{\partial^{m_{k}+1+\sum_{l \in \mathcal{K} \backslash\{k\}} m_{l} \phi(\sqrt{\tau}(\mathbf{x}-\beta))}}{\partial \beta_{k}^{m_{k}+1} \prod_{l \in \mathcal{K} \backslash\{k\}} \partial \beta_{l}^{m_{l}}} \mathrm{~d} \mathbf{x}\right| \\
& \quad<\epsilon_{k} \int_{\mathbb{R}^{K}}\left|f(\mathbf{x}) g_{m_{k}+2}\left(\sqrt{\tau}\left(x_{k}-\beta_{k}-\gamma \rho z_{k}\right)\right) \prod_{l \in \mathcal{K} \backslash\{k\}} g_{m_{l}}\left(\sqrt{\tau}\left(x_{l}-\beta_{l}\right)\right)\right| \mathrm{d} \mathbf{x}
\end{aligned}
$$

Step 2(ii). Recall now (13) above. For any $x_{k} \in \mathbb{R} \backslash\left(-\delta_{k}, \delta_{k}\right)$ we have

$$
\left|x_{k}-\beta_{k}-\gamma \rho z_{k}\right| \geq\left|x_{k}-\beta_{k}\right|-\gamma \rho\left|z_{k}\right|>\left|x_{k}-\beta_{k}\right|-1 \geq\left|x_{k}\right|-\left|\beta_{k}\right|-1 \geq 0
$$


so that

$$
\begin{aligned}
\phi\left(\sqrt{\tau(1-\alpha)}\left(x_{k}-\beta_{k}-\gamma \rho z_{k}\right)\right) & <\phi\left(\sqrt{\tau(1-\alpha)}\left(\left|x_{k}-\beta_{k}\right|-1\right)\right) \\
& =e^{\tau(1-\alpha)\left(\left|x_{k}-\beta_{k}\right|-\frac{1}{2}\right)} \phi\left(\sqrt{\tau(1-\alpha)}\left(x_{k}-\beta_{k}\right)\right) \\
& =e^{\tau(1-\alpha)\left(x_{k} \beta_{k}+\left|x_{k}-\beta_{k}\right|-\frac{1+\beta_{k}^{2}}{2}\right)} \phi\left(\sqrt{\tau(1-\alpha)} x_{k}\right) \\
& <e^{\tau(1-\alpha)\left(x_{k} \beta_{k}+\left|x_{k}-\beta_{k}\right|\right)} \phi\left(\sqrt{\tau(1-\alpha)} x_{k}\right) \\
& \leq e^{\tau(1-\alpha)\left(\left|x_{k} \beta_{k}\right|+\left|x_{k}\right|+\left|\beta_{k}\right|\right)} \phi\left(\sqrt{\tau(1-\alpha)} x_{k}\right) \\
& \leq e^{\tau(1-\alpha)\left(\left|x_{k}\right|+2\left|\beta_{k}\right|\right)} \phi\left(\sqrt{\tau(1-\alpha)} x_{k}\right)
\end{aligned}
$$

Clearly, the first two terms of the integrand in (15) give

$$
\begin{aligned}
& \left|f(\mathbf{x}) g_{m_{k}+2}\left(\sqrt{\tau}\left(x_{k}-\beta_{k}-\gamma \rho z_{k}\right)\right)\right| \\
& \quad<C_{k}^{\prime}|f(\mathbf{x})| e^{\tau(1-\alpha)\left|x_{k}\right|} \phi\left(\sqrt{\tau(1-\alpha)} x_{k}\right) \quad \forall x_{k} \in \mathbb{R} \backslash \mathcal{B}_{\mathbf{0}}\left(\delta_{k}\right)
\end{aligned}
$$

where $C_{k}^{\prime}=\sqrt{\tau^{m_{k}+2}} C_{m_{k}+2} e^{2 \tau(1-\alpha)\left|\beta_{k}\right|}$. By hypothesis, however, there exist $r \in(0, \tau / 2)$ and $C>0$ such that $|f(\mathbf{x})| \leq C e^{r|\mathbf{x}|^{2}}$ on $\mathbb{R}^{K}$. Moreover, by Lemma A.1 above, for any $r_{k}>0$, we can find $C_{k}^{\prime \prime}>0$ such that $e^{\tau(1-\alpha)\left|x_{k}\right|}<C_{k}^{\prime \prime} e^{r_{k} x_{k}^{2}}$. Choosing, thus, $r_{k} \in(0, \tau / 2-r)$ and $\alpha \in\left(0, \frac{\tau-2\left(r+r_{k}\right)}{\tau}\right)$ Lemma A.2 ensures the existence of $r^{\prime \prime}, \delta_{0}^{\prime \prime}>0$ such that

$$
\left|f(\mathbf{x}) g_{m_{k}+2}\left(\sqrt{\tau}\left(x_{k}-\beta_{k}-\gamma \rho z_{k}\right)\right)\right|<C C_{k}^{\prime} C_{k}^{\prime \prime} e^{r\left|\mathbf{x}_{-k}\right|^{2}} \phi\left(\sqrt{r^{\prime \prime}} x_{k}\right) \quad \forall x_{k} \in \mathbb{R} \backslash \mathcal{B}_{\mathbf{0}}\left(\max \left\{\delta_{k}, \delta_{0}^{\prime \prime}\right\}\right)
$$

We have just established that, viewed as a function of $z_{k}$, the integrand in (15) is bounded above on $\mathbb{R}^{K} \backslash \mathcal{B}_{\mathbf{0}}\left(\max \left\{\delta_{k}, \delta_{0}^{\prime \prime}\right\}\right)$ by a function that is independent of $z_{k}$ and integrable on $\mathbb{R}^{K} \backslash \mathcal{B}_{\mathbf{0}}\left(\max \left\{\delta_{k}, \delta_{0}^{\prime \prime}\right\}\right)$.

Regarding the interior of the left-out neighbourhood, observe that

$$
\left|x_{k}-\beta_{k}-\gamma \rho z_{k}\right| \leq\left|x_{k}-\beta_{k}\right|+\left|\gamma \rho z_{k}\right|<\left|x_{k}-\beta_{k}\right|+1
$$

and, thus,

$$
\begin{aligned}
\left|g_{m_{k}+2}\left(\sqrt{\tau}\left(x_{k}-\beta_{k}-\gamma \rho z_{k}\right)\right)\right| & =\phi\left(\sqrt{\tau}\left(x_{k}-\beta_{k}-\gamma \rho z_{k}\right)\right)\left|H_{m_{k}+2}\left(\sqrt{\tau}\left(x_{k}-\beta_{k}-\gamma \rho z_{k}\right)\right)\right| \\
& <\left|H_{m_{k}+2}\left(\sqrt{\tau}\left(x_{k}-\beta_{k}-\gamma \rho z_{k}\right)\right)\right| \\
& \leq \sum_{i=0}^{m_{k}+2}\left|c_{i}\right| \tau^{i / 2}\left|x_{k}-\beta_{k}-\gamma \rho z_{k}\right|^{i} \\
& <\sum_{i=0}^{m_{k}+2}\left|c_{i}\right| \tau^{i / 2}\left(\left|x_{k}-\beta_{k}\right|+1\right)^{i} \\
& <\sum_{i=0}^{m_{k}+2}\left|c_{i}\right| \tau^{i / 2} e^{i\left(\left|x_{k}\right|+\left|\beta_{k}\right|+1\right)}=C_{0}^{\prime \prime} e^{\left(m_{k}+2\right)\left|x_{k}\right|}
\end{aligned}
$$

where $C_{0}^{\prime \prime}=e^{\left(m_{k}+2\right)\left(\left|\beta_{k}\right|+1\right)} \sum_{i=0}^{m_{k}+2}\left|c_{i}\right| \tau^{i / 2}$. Clearly, also on $\mathcal{B}_{\mathbf{0}}\left(\max \left\{\delta_{k}, \delta_{0}^{\prime \prime}\right\}\right)$, as a function of $z_{k}$, the integrand in (15) is bounded above by an integrable function that is independent of $z_{k}$. 
Given these obsevations, and as $g_{m_{k}+2}\left(\sqrt{\tau}\left(x_{k}-\beta_{k}-\gamma \rho z_{k}\right)\right)$ is continuous in $z_{k}$ for any $x_{k} \in \mathbb{R}$, Lemma A.3 above ensures that the continuity in $z_{k}$ on $\left(-\epsilon_{k}, \epsilon_{k}\right)$ is preserved under the integral sign in (15). That is,

$$
\begin{aligned}
& \lim _{\left|z_{k}\right| \rightarrow 0} \int_{\mathbb{R}^{K}}\left|f(\mathbf{x}) g_{m_{k}+2}\left(\sqrt{\tau}\left(x_{k}-\beta_{k}-\gamma \rho z_{k}\right)\right) \prod_{l \in \mathcal{K} \backslash\{k\}} g_{m_{l}}\left(\sqrt{\tau}\left(x_{l}-\beta_{l}\right)\right)\right| \mathrm{d} \mathbf{x} \\
& =\int_{\mathbb{R}^{K}}\left|f(\mathbf{x}) g_{m_{k}+2}\left(\sqrt{\tau}\left(x_{k}-\beta_{k}\right)\right) \prod_{l \in \mathcal{K} \backslash\{k\}} g_{m_{l}}\left(\sqrt{\tau}\left(x_{l}-\beta_{l}\right)\right)\right| \mathrm{d} \mathbf{x}
\end{aligned}
$$

Step 2(iii). To establish now the required differentiability, it suffices to show that the integral on the right-hand side of (17) above is well defined. To this end, recall once more (13). Letting $\tilde{z}_{k}=0$, we have

$$
\left|\prod_{l \in \mathcal{K} \backslash\{k\}} g_{m_{l}}\left(\sqrt{\tau}\left(x_{l}-\beta_{l}\right)\right) g_{m_{k}+2}\left(\sqrt{\tau}\left(x_{k}-\beta_{k}\right)\right)\right| \leq C_{3}^{\prime} \phi(\sqrt{\tau(1-\alpha)}(\mathbf{x}-\beta))
$$

for any $\mathbf{x} \in \mathbb{R}^{K} \backslash \mathcal{B}_{\mathbf{0}}\left(\delta_{0}\right)$ and where $C_{0}^{\prime \prime \prime}=C_{m_{k}+2} \prod_{l \in \mathcal{K} \backslash\{k\}} C_{m_{l}}$. Choosing though $\alpha$ as in Step 1(iii) we have that

$$
|f(\mathbf{x}) \phi(\sqrt{\tau(1-\alpha)}(\mathbf{x}-\beta))| \leq C \phi\left(\sqrt{r^{\prime}} \mathbf{x}\right) \quad \forall \mathbf{x} \in \mathbb{R}^{K} \backslash \mathcal{B}_{\mathbf{0}}\left(\delta_{0}^{\prime}\right)
$$

Clearly, the integrand on the right-hand side of (17) is bounded above on $\mathbb{R}^{K} \backslash \mathcal{B}_{\mathbf{0}}\left(\max \left\{\delta_{0}, \delta_{0}^{\prime}\right\}\right)$ by the function $C C_{0}^{\prime \prime \prime} \phi\left(\sqrt{r^{\prime}} \mathbf{x}\right)$; hence, its integral is well defined. To complete the argument, let $\epsilon_{k} \rightarrow 0$ in (15).

Step 3. To establish finally continuous differentiability, it suffices to show that the partial derivatives are continuous. And as $\mathbf{m}$ above was taken arbitrarily, it suffices in turn to show that $F^{\mathbf{m}}(\cdot)$ itself is continuous. Taking $\epsilon \in(0,1)$, consider then the neighborouhood $\mathcal{B}_{\beta}(\epsilon)$. For any $\mathbf{z} \in \mathcal{B}_{\beta}(\epsilon)$, using again (13) above and the mean-value theorem, we have

$$
\begin{aligned}
& \left|F^{(\mathbf{m})}(\beta+\mathbf{h})-F^{(\mathbf{m})}(\beta)\right| \\
& \quad \leq \int_{\mathbb{R}^{K}}|f(\mathbf{x})| \prod_{k=1}^{K}\left|g_{m_{k}}\left(\sqrt{\tau}\left(x_{k}-\beta_{k}-h_{k}\right)\right)-g_{m_{k}}\left(\sqrt{\tau}\left(x_{k}-\beta_{k}\right)\right)\right| \mathrm{d} \mathbf{x} \\
& \quad=\left(\prod_{k=1}^{K}\left|h_{k}\right|\right) \int_{\mathbb{R}^{K}}|f(\mathbf{x})| \prod_{k=1}^{K}\left|g_{m_{k+1}}\left(\sqrt{\tau}\left(x_{k}-\beta_{k}-\gamma_{k} h_{k}\right)\right)\right| \mathrm{d} \mathbf{x} \\
& \quad<\epsilon^{K} \int_{\mathbb{R}^{K}}|f(\mathbf{x})| \prod_{k=1}^{K}\left|g_{m_{k+1}}\left(\sqrt{\tau}\left(x_{k}-\beta_{k}-\gamma_{k} h_{k}\right)\right)\right| \mathrm{d} \mathbf{x}
\end{aligned}
$$

where $\gamma_{k} \in(0,1)$ for $k \in \mathcal{K}$. The remainder of the argument is trivially similar to that in steps 2(ii)-(iii) above.

Lemma A.5 Let $(\tau, \beta) \in \mathbb{R}_{++} \times \mathbb{R}^{K}$ be a parameter vector. Suppose also that the continuously-differentiable function $f: \mathbb{R}^{K} \rightarrow \mathbb{R}$ satisfies

$$
|f(\mathbf{x})| \leq C e^{r|\mathbf{x}|^{2}} \quad \forall \mathbf{x} \in \mathbb{R}^{K}
$$


for some constants $(r, C) \in(0, \tau / 2) \times \mathbb{R}_{++}$. The $\mathbb{R}^{K} \rightarrow \mathbb{R}$ function

$$
F(\beta):=\int_{\mathbb{R}^{K}} f(\beta+\mathbf{x} / \sqrt{\tau}) \phi(\mathbf{x}) d \mathbf{x}
$$

is well defined and $C^{1}$, with its partial derivatives given by

$$
D_{k} F(\beta)=\int_{\mathbb{R}^{K}} D_{k} f(\beta+\mathbf{x} / \sqrt{\tau}) \phi(\mathbf{x}) d \mathbf{x}, \quad k \in \mathcal{K}
$$

Proof By changing the variables of integration, we have

$$
F(\beta)=\sqrt{\tau} \int_{\mathbb{R}^{K}} f(\mathbf{z}) \phi(\sqrt{\tau}(\mathbf{z}-\beta)) \mathrm{d} \mathbf{z}
$$

That $F(\cdot)$ is well defined and $C^{1}$ follows from Lemma A.4. Moreover, its partial derivatives are given by

$D_{k} F(\beta)=\sqrt{\tau} \int_{\mathbb{R}^{K}} f(\mathbf{z}) \frac{\partial}{\partial \beta_{k}} \phi(\sqrt{\tau}(\mathbf{z}-\beta)) \mathrm{d} \mathbf{z}=-\sqrt{\tau} \int_{\mathbb{R}^{K}} f(\mathbf{z}) \frac{\partial}{\partial z_{k}} \phi(\sqrt{\tau}(\mathbf{z}-\beta)) \mathrm{d} \mathbf{z}, \quad k \in \mathcal{K}$

To establish the expession in the claim, fix first an arbitrary $\mathbf{z}_{-k} \in \mathbb{R}^{K-1}$. By Lemma A.2 above, we can find $r_{0}, \delta_{0}>0$ such that

$$
\begin{aligned}
f(\mathbf{x}) \phi(\sqrt{\tau}(\mathbf{z}-\beta)) \leq C e^{r|\mathbf{z}|^{2}-\frac{\tau}{2}|\mathbf{z}-\beta|^{2}} & =C e^{r\left|\mathbf{z}_{-k}\right|^{2}-\tau\left|\mathbf{z}_{-k}-\beta_{-k}\right|^{2}} e^{r z_{k}^{2}-\frac{\tau}{2}\left(z_{k}-\beta_{k}\right)^{2}} \\
& \leq C e^{r\left|\mathbf{z}_{-k}\right|^{2}-\frac{\tau}{2}\left|\mathbf{z}_{-k}-\beta-_{-k}\right|^{2}} \phi\left(\sqrt{2 r_{0}} z_{k}\right) \quad \forall z_{k} \in \mathbb{R} \backslash \mathcal{B}_{\mathbf{0}}\left(\delta_{0}\right)
\end{aligned}
$$

This implies that

$$
\lim _{x_{k} \rightarrow \pm \infty}|f(\mathbf{z}) \phi(\sqrt{\tau}(\mathbf{z}-\beta))| \leq \lim _{z_{k} \rightarrow \pm \infty} C e^{r\left|\mathbf{z}_{-k}\right|^{2}-\frac{\tau}{2}\left|\mathbf{z}_{-k}-\beta_{-k}\right|^{2}} \phi\left(\sqrt{2 r_{0}} \mathbf{z}\right)=0
$$

and, thus, integration by parts establishes that

$$
-\int_{\mathbb{R}} f(\mathbf{z}) \frac{\partial}{\partial z_{k}} \phi(\sqrt{\tau}(\mathbf{z}-\beta)) \mathrm{d} z_{k}=\int_{\mathbb{R}} \phi(\sqrt{\tau}(\mathbf{z}-\beta)) \frac{\partial}{\partial z_{k}} f(\mathbf{z}) \mathrm{d} z_{k}
$$

Integrating now over the remaining $K-1$ dimensions, we get that

$$
\begin{aligned}
D_{k} F(\beta)=-\sqrt{\tau} \int_{\mathbb{R}^{K}} f(\mathbf{z}) \frac{\partial}{\partial z_{k}} \phi(\sqrt{\tau}(\mathbf{z}-\beta)) \mathrm{d} \mathbf{z} & =\sqrt{\tau} \int_{\mathbb{R}^{K}} \phi(\sqrt{\tau}(\mathbf{z}-\beta)) \frac{\partial}{\partial z_{k}} f(\mathbf{z}) \mathrm{d} \mathbf{z} \\
& =\sqrt{\tau} \int_{\mathbb{R}^{K}} \phi(\mathbf{x}) \frac{\partial}{\partial x_{k}} f(\beta+\mathbf{x} / \sqrt{\tau}) \mathrm{d} \mathbf{x} \\
& =\int_{\mathbb{R}^{K}} \phi(\mathbf{x}) \frac{\partial}{\partial \beta_{k}} f(\beta+\mathbf{x} / \sqrt{\tau}) \mathrm{d} \mathbf{x}
\end{aligned}
$$

with the third equality above due changing once again the variables of integration.

Lemma A.6 Given $(T, \beta) \in \mathbb{R}_{++} \times \mathbb{R}^{K}$, let the function $f:[0, T] \times \mathbb{R}^{K} \rightarrow \mathbb{R}$ be continuous. Suppose also that the latter satisfies

$$
|f(s, \mathbf{x})| \leq C_{0} e^{r_{0}|\mathbf{x}|^{2}} \quad \forall(s, \mathbf{x}) \in(0, T] \times \mathbb{R}^{K}
$$

for some constants $\left(C_{0}, r_{0}\right) \in \mathbb{R}_{++} \times\left(0, \frac{1}{2 T}\right)$. There exist then constants $C, r \in \mathbb{R}_{++}$such that

$$
|f(s, \beta+\sqrt{s-t} \mathbf{x})| \phi(\mathbf{x})<C \phi(\sqrt{r} \mathbf{x}) \quad \forall(t, s, \mathbf{x}) \in[0, T) \times(t, T] \times \mathbb{R}^{K}
$$


Proof Under the given growth condition, for any $(t, s, \mathbf{x}) \in[0, T) \times(t, T] \times \mathbb{R}^{K}$, we have

$$
\begin{aligned}
|f(s, \beta+\sqrt{s-t} \mathbf{x})| \phi(\mathbf{x}) & \leq C_{0} \phi(\mathbf{x}) e^{r_{0}|\beta+\sqrt{s-t} \mathbf{x}|^{2}} \\
& =C_{0} \phi(\mathbf{x}) e^{r_{0}\left(|\beta|^{2}+(s-t)|\mathbf{x}|^{2}+2 \sqrt{s-t} \beta^{\top} \mathbf{x}\right)} \\
& \leq C_{0} \phi(\mathbf{x}) e^{r_{0}\left(|\beta|^{2}+(T-t)|\mathbf{x}|^{2}+2 \sqrt{T-t}|\beta||\mathbf{x}|\right)} \\
& <C_{0} \phi(\mathbf{x}) e^{r_{0}\left(|\beta|^{2}+T|\mathbf{x}|^{2}+2 \sqrt{T}|\beta||\mathbf{x}|\right)} \\
& =\frac{C_{0}}{\sqrt{2 \pi}} e^{r_{0}\left(|\beta|^{2}+2 \sqrt{T}|\beta||\mathbf{x}|\right)-\left(\frac{1}{2}-r_{0} T\right)|\mathbf{x}|^{2}}
\end{aligned}
$$

Recall now Lemma A.1 above. We can find constants $C_{1} \in \mathbb{R}_{++}$and $r_{1} \in\left(0, \frac{1}{2}-r_{0} T\right)$ such that

$$
e^{2 r_{0} \sqrt{T}|\beta||\mathbf{x}|}<C_{1} e^{r_{1}|\mathbf{x}|^{2}} \quad \forall \mathbf{x} \in \mathbb{R}^{K}
$$

The claim follows by setting $r=1-2\left(r_{0} T-r_{1}\right)$ and $C=C_{0} C_{1} e^{r_{0}|\beta|^{2}} / \sqrt{2 \pi}$.

Proposition A.1 Let $\mathcal{T}=[0, T]$ for some $T \in \mathbb{R}_{++}$and suppose that the price process is given by (2) in the main text with condition A.1(i) satisfied. Then, for any $(j, n, k) \in$ $\mathcal{K} \cup\{0\} \times \mathcal{K} \times \mathcal{K}$, the following hold

(i) $\lim _{t \rightarrow T}\left(m P_{j}\right)(\mathcal{I}(\omega, t))=M_{j}(\mathcal{I}(\omega, T))$ almost everywhere (a.e.) on $\Omega$.

(ii) $\lim _{t \rightarrow T} D_{k}\left(m P_{j}\right)(\mathcal{I}(\omega, t))=D_{k} M_{j}(\mathcal{I}(\omega, T))$ a.e. on $\Omega$.

(iii) $\lim _{t \rightarrow T} D_{k} p_{n}(\mathcal{I}(\omega, t))=D_{k} G_{n / 0}(\mathcal{I}(\omega, T))$ a.e. on $\left\{\omega \in \Omega: \beta(\omega, T) \in S_{G_{0}}\right\}$.

Proof (i). By Lemma A.4, $\left(m P_{1 j}\right)(t, \beta):=\int_{\mathbb{R}^{K}} M_{j}(T, \beta+\sqrt{T-t} \mathbf{x}) \phi(\mathbf{x}) \mathrm{d} \mathbf{x}$ is well defined at any $(t, \beta) \in[0, T) \times \mathbb{R}^{K}$. Moreover, $M_{j}(T, \cdot)$ being continuous so is the function $t \rightarrow M_{j}(T, \beta+\sqrt{T-t} \mathbf{x})$ everywhere on $(0, T)$ for any given $(\beta, \mathbf{x}) \in \mathbb{R}^{K} \times \mathbb{R}^{K}$. Furthermore, by Lemma A.6, there exist constants $r, C>0$ such that

$$
\left|M_{j}(T, \beta+\sqrt{T-t} \mathbf{x})\right| \phi(\mathbf{x})<C \phi(\sqrt{r} \mathbf{x}) \quad \forall(t, \mathbf{x}) \in(0, T) \times \mathbb{R}^{K}
$$

By Lemma A.3 then the preceding observations mean that, for any given $\beta \in \mathbb{R}^{K}$, $\left(m P_{1 j}\right)(t, \beta)$ is continuous in $t$ everywhere on $(0, T)$.

Taking now any $\epsilon \in\left(0, \frac{1}{2 r}-T\right)$, the argument above remains valid if $T$ is replaced by $T+\epsilon$. That is, the function $t \rightarrow\left(m P_{1 j}\right)(t, \beta)$ is continuous also on $(0, T+\epsilon)$. It is therefore leftcontinuous at $T$ in the original problem and thus

$$
\begin{aligned}
\lim _{t \rightarrow T}\left(m P_{1 j}\right)(t, \beta) & =\lim _{t \rightarrow T} \int_{\mathbb{R}^{K}} M_{j}(T, \beta+\sqrt{T-t} \mathbf{x}) \phi(\mathbf{x}) \mathrm{d} \mathbf{x} \\
& =\int_{\mathbb{R}^{K}} M_{j}(T, \beta) \phi(\mathbf{x}) \mathrm{d} \mathbf{x}=M_{j}(T, \beta)
\end{aligned}
$$

(ii) Replacing the function $M_{j}(T, \cdot)$ with $D_{k} M_{j}(T, \cdot)$ in the preceding argument, we obtain also that $\lim _{t \rightarrow T} D_{k}\left(m P_{j}\right)(t, \beta)=D_{k} M_{j}(T, \beta)$.

(iii) Since $p_{n}(\cdot)=\left(m P_{n}\right)(\cdot) /\left(m P_{0}\right)(\cdot)$, by (i)-(ii) above we get that

$$
\begin{aligned}
M_{0}(T, \beta)^{2} \lim _{t \rightarrow T} D_{k} p_{n}(t, \beta) & =\lim _{t \rightarrow T}\left(m P_{0}\right)(t, \beta)^{2} D_{k} p_{n}(t, \beta) \\
& =\lim _{t \rightarrow T}\left(m P_{0}\right)(t, \beta) D_{k}\left(m P_{n}\right)(t, \beta)-\lim _{t \rightarrow T}\left(m P_{n}\right)(t, \beta) D_{k}\left(m P_{0}\right)(t, \beta) \\
& =M_{0}(T, \beta) D_{k} M_{n}(T, \beta)-M_{n}(T, \beta) D_{k} M_{0}(T, \beta)
\end{aligned}
$$


As long as $\beta \in S_{G_{0}}$, therefore, we have

$$
\lim _{t \rightarrow T} D_{k} p_{n}(t, \beta)=D_{k}\left(M_{n} / M_{0}\right)(T, \beta)=D_{k} G_{n / 0}(T, \beta)
$$

To complete the argument, recall that the Brownian paths $\{\beta(\omega, s): s \in(0, T)\}$ are continuous almost surely (with respect to $\pi$ ) on $\Omega$. For almost all $\omega \in \Omega$ that is $\beta(\omega, t)$ approaches $\beta(\omega, T)$ as $t \rightarrow T$.

Lemma A.7 Suppose $f: \mathbb{R}^{K} \rightarrow \mathbb{R}$ is measurable on $\mathbb{R}^{K}$ and satisfies ${ }^{23}$

$$
\exists C>0, r \in\left(0, \frac{1}{2 T}\right):|f(\mathbf{x})| \leq C e^{r|\mathbf{x}|^{2}} \quad \forall \mathbf{x} \in \mathbb{R}^{K}
$$

Let also $\beta$ be a standard K-dimensional Brownian motion and

$$
F(t, \beta)=\mathbb{E}\left[f\left(\beta_{T}\right) \mid \beta_{t}=\beta\right]
$$

Then $F(\cdot)$ is an analytic function of $(t, \beta)$ on $(0, T) \times \mathbb{R}^{K}$.

Proof The proof proceeds in exactly the same way as in that of Theorem B.4 in Anderson and Raimondo [2], but for a trivial adaptation of the argument that supports equations (23)-(27) in that paper. Specifically, fixing $t<T$, Eq. (21) in Anderson and Raimondo [2] gives

$$
\begin{aligned}
F(t, \beta) & =(2 \pi(T-t))^{-K / 2} \int_{\mathbb{R}^{K}} f(\beta+\mathbf{x}) e^{-\frac{|\mathbf{x}|^{2}}{2(T-t)}} \mathrm{d} \mathbf{x} \\
& =\frac{e^{-\frac{|\beta|^{2}}{2(T-t)}}}{(2 \pi(T-t))^{K / 2}} \int_{\mathbb{R}^{K}} \sum_{k=0}^{+\infty} \frac{1}{(T-t)^{k}} \sum_{k_{1}+\cdots+k_{K}=k} \frac{\left(\beta_{1} y_{1}\right)^{k_{1}} \cdots\left(\beta_{K} y_{K}\right)^{k_{K}}}{k_{1} ! \cdots k_{K} !} f(\mathbf{y}) e^{-\frac{|\mathbf{y}|^{2}}{2(T-t)}} \mathrm{d} \mathbf{y}
\end{aligned}
$$

Under the present growth condition, however, Eqs. (22)-(27) in Anderson and Raimondo [2] can be replaced now by the following steps

$$
\begin{aligned}
& \left|\frac{1}{(2 \pi(T-t))^{K / 2}} \int_{\mathbb{R}^{K}} \frac{y_{1}^{k_{1}} \cdots y_{K}^{k_{K}}}{k_{1} ! \cdots k_{K} !} f(\mathbf{y}) e^{-\frac{|\mathbf{y}|^{2}}{2(T-t)}} \mathrm{d} \mathbf{y}\right| \\
& \quad \leq \frac{1}{(2 \pi(T-t))^{K / 2}} \int_{\mathbb{R}^{K}} \frac{\left|y_{1}\right|^{k_{1}} \cdots\left|y_{K}\right|^{k_{K}}}{k_{1} ! \cdots k_{K} !}|f(\mathbf{y})| e^{-\frac{|\mathbf{y}|^{2}}{2(T-t)}} \mathrm{d} \mathbf{y} \\
& \leq \frac{C}{(2 \pi(T-t))^{K / 2}} \int_{\mathbb{R}^{K}} \frac{\left|y_{1}\right|^{k_{1}} \cdots\left|y_{K}\right|^{k_{K}}}{k_{1} ! \cdots k_{K} !} e^{-\frac{\left.(1-2 r(T-t)) \mathbf{y}\right|^{2}}{2(T-t)}} \mathrm{d} \mathbf{y} \\
& \leq \frac{C}{\sqrt{k_{1} ! \cdots k_{K} !}}\left(\frac{T-t}{1-2 r(T-t)}\right)^{K / 2} \leq C \frac{k_{1} ! \cdots k_{K} !}{\sqrt{\frac{1}{T-t}-2 r}}
\end{aligned}
$$

where the second inequality above follows from the growth condition itself and the third from the formula for the $k_{i}$ th moment of the absolute value of a normal random variable. Clearly, the last inequality above provides an upper bound for the absolute value of the coefficient of $\beta_{1}^{k_{1}} \cdots \beta_{K}^{k_{K}}$ in the power series of equation (27) in Anderson and Raimondo [2]. Hence, the power series in question converges also here absolutely within a positive radius of convergence (see Proposition 2.2.10 in Krantz and Parks [20]). The remainder of the proof is identical to that in Anderson and Raimondo [2].

23 This result is an adaptation of Theorem B.4 in Anderson and Raimondo [2]. The latter assumes the growth condition in Lemma A.1 above, which is stronger than the one in the present statement. 


\section{B Price dynamics for dividend flows}

Restricting first attention to the time-horizon being finite $\left(\mathcal{T}=[0, T]\right.$ for some $\left.T \in \mathbb{R}_{++}\right)$, recall that the Brownian paths $\{\beta(\omega, s): s \in(0, T)\}$ are continuous almost surely with respect to $\pi$. Hence, for any function $f:[0, T] \times \mathbb{R}^{K} \rightarrow \mathbb{R}$ that is continuous on $(0, T) \times \mathbb{R}^{K}$ the paths $\{f(\mathcal{I}(\omega, s))\}_{s \in(0, T)}$ will be continuous almost everywhere on $\Omega$. For almost all such paths and for any $t \in[0, T)$, therefore, the time-integral can be approximated as a Riemann-Stieltjes sum:

$$
\begin{aligned}
\int_{t}^{T} f(\mathcal{I}(\omega, s)) \mathrm{d} s & =\lim _{\Pi_{\tau} \rightarrow 0} \sum_{i=1}^{\tau} f\left(\mathcal{I}\left(\omega, s_{i-1}\right)\right) \Delta_{i} \\
& =\lim _{\Pi_{\tau} \rightarrow 0} \sum_{i=1}^{\tau} f\left(s_{i-1}, \beta\left(\omega, s_{i-1}\right)\right) \Delta_{i} \\
& =\lim _{\Pi_{\tau} \rightarrow 0} \sum_{i=1}^{\tau} f\left(s_{i-1}, \beta(\omega, t)+\sum_{j=0}^{i-1} \beta\left(\omega, s_{j+1}\right)-\beta\left(\omega, s_{j}\right)\right) \Delta_{i}
\end{aligned}
$$

where $\Pi_{\tau}=\max _{i=1, \ldots, \tau}\left\{\Delta_{i}=s_{i}-s_{i-1}\right\}$ denotes the mesh of the typical partition $t=$ $s_{0}<s_{1}<\cdots<s_{\tau-1}=T$ for some $\tau \in \mathbb{N} \backslash\{0\}$ in the approximating sequence. For any $s_{j}, s_{j+1} \in[t, T]$ though the increments $\beta\left(\omega, s_{j+1}\right)-\beta\left(\omega, s_{j}\right)$ are independent of $\mathcal{F}_{t}$ and i.i.d $\mathcal{N}\left(\mathbf{0}^{K},\left(s_{j+1}-s_{j}\right) \mathrm{I}_{K}\right)$. Letting therefore $\mathbf{x}_{j} \sim \mathcal{N}\left(\mathbf{0}^{K}, \Delta_{j+1} \mathrm{I}_{K}\right)$ and thus $\sum_{j=0}^{i-1} \mathbf{x}_{j} \sim$ $\mathcal{N}\left(\mathbf{0}^{K},\left(s_{i-1}-t\right) \mathrm{I}_{K}\right)$, it follows that $f\left(s_{i-1}, \beta_{s_{i-1}}\right) \sim_{\mid \beta_{t}=\beta} f\left(s_{i-1}, \beta+\sqrt{s_{i-1}-t} \mathbf{x}\right)$; that is,

$\mathbb{E}_{\pi}\left[f(\mathcal{I}(\omega, s)) \mid \mathcal{F}_{t}\right]=\mathbb{E}_{\pi}\left[f\left(s, \beta_{s}\right) \mid \beta_{t}=\beta\right]=\mathbb{E}_{\mathbf{x}}[f(s, \beta+\sqrt{s-t} \mathbf{x})] \quad(s, \beta) \in(t, T) \times \mathbb{R}^{K}$ where $\sim_{\mid \beta_{t}=\beta}$ denotes distribution conditional on $\beta_{t}=\beta \in \mathbb{R}^{K}$ while $\mathbf{x} \sim \mathcal{N}\left(\mathbf{0}^{K}, \mathrm{I}_{K}\right)$. By Fubini's theorem then (see, for instance, Corollary 13.9 in Schilling [29]), as long as the quantity $\int_{t}^{T} \mathbb{E}_{\pi}\left[f(\mathcal{I}(\omega, s)) \mid \mathcal{F}_{t}\right] \mathrm{d} s$ is well defined, it cannot but be

$$
\begin{aligned}
\mathbb{E}_{\pi}\left[\int_{t}^{T} f(\mathcal{I}(\omega, s)) \mathrm{d} s \mid \mathcal{F}_{t}\right] & =\int_{t}^{T} \mathbb{E}_{\pi}\left[f(\mathcal{I}(\omega, s)) \mid \mathcal{F}_{t}\right] \mathrm{d} s \\
& =\int_{t}^{T} \mathbb{E}_{\pi}\left[f\left(s, \beta_{s}\right) \mid \beta_{t}=\beta\right] \mathrm{d} s \\
& =\int_{t}^{T} \mathbb{E}_{\mathbf{x}}[f(s, \beta+\sqrt{s-t} \mathbf{x})] \mathrm{d} s \quad(t, \beta) \in[0, T) \times \mathbb{R}^{K}
\end{aligned}
$$

Recall now Assumption A1(ii). As $r<\frac{1}{2 T}<\frac{1}{2(T-t)}<\frac{1}{2(s-t)} \forall s \in(t, T)$, it follows by Lemma A.4 above that the quantity $\mathbb{E}_{\mathbf{x}}\left[m_{j}(s, \beta+\sqrt{s-t} \mathbf{x})\right]$ is well defined at any $(s, \beta) \in[t, T] \times \mathbb{R}^{K}$. By the following result, moreover, it is also integrable on $(t, T)$.

Lemma B.1 Given $T \in \mathbb{R}_{++}$and $(t, \beta) \in[0, T) \times \mathbb{R}^{K}$, let the function $f:(0, T) \times \mathbb{R}^{K} \rightarrow \mathbb{R}$ be continuous and satisfy

$$
|f(s, \mathbf{x})| \leq C_{0} e^{r_{0}|\mathbf{x}|^{2}} \quad \forall(s, \mathbf{x}) \in(0, T) \times \mathbb{R}^{K}
$$

for some constants $\left(C_{0}, r_{0}\right) \in \mathbb{R}_{++} \times\left(0, \frac{1}{2 T}\right)$. If $\mathbf{x} \sim \mathcal{N}\left(\mathbf{0}^{K}, I_{K}\right)$ then $\mathbb{E}_{\mathbf{x}}[f(s, \beta+\sqrt{s-t} \mathbf{x})]$ is integrable on $(t, T)$. 
Proof Since $r_{0}<\frac{1}{2 T}<\frac{1}{2(T-t)}<\frac{1}{2(s-t)} \forall s \in(t, T)$, it follows from Lemma A.4 (for $\mathbf{m}=\mathbf{0}^{K}$ ) that

$$
\mathbb{E}_{\mathbf{x}}[f(s, \beta+\sqrt{s-t} \mathbf{x})]=\frac{1}{\sqrt{s-t}} \int_{\mathbb{R}^{K}} f(s, \mathbf{x}) \phi\left(\frac{\mathbf{x}-\beta}{\sqrt{s-t}}\right) \mathrm{d} \mathbf{x}
$$

is well defined on $(t, T)$. By Lemma A.6, moreover, there exist a constant $r>0$ and a continuous function $C: \mathbb{R}^{K} \rightarrow \mathbb{R}_{++}$such that

$$
\int_{t}^{T} \mathbb{E}_{\mathbf{x}}[|f(s, \beta+\sqrt{s-t} \mathbf{x})|] \mathrm{d} s<C(\beta) \int_{t}^{T} \int_{\mathbb{R}^{K}} e^{-\frac{r|\mathbf{x}|^{2}}{2}} \mathrm{~d} \mathbf{x} \mathrm{d} s=(T-t) C(\beta) \sqrt{2 \pi / r}
$$

The claim follows.

The preceding discussion establishes that the expressions in (5)-(6) in the main text follow from (19)-(20), and are indeed well defined.

With respect now to (7) in the main text, by Lemma A.5, at any $s \in(t, T]$ the integrand in (6) has a well-defined partial derivative: $D_{k} \mathbb{E}_{\mathbf{x}}\left[m_{j}(s, \beta+\sqrt{s-t} \mathbf{x})\right]=$ $\mathbb{E}_{\mathbf{x}}\left[D_{k} m_{j}(s, \beta+\sqrt{s-t} \mathbf{x})\right]$. Given then Assumption A1(ii), Lemma A.4 ensures that the partial derivative is continuous in $\beta$ for any given $(t, s) \in(0 . T) \times(t . T)$. Moreover, we can apply again here the argument in the proof of Proposition A.1(i) to establish that, for any given $(t, \beta) \in[0 . T) \times \mathbb{R}^{K}$, the partial derivative is also continuous in $s$ everywhere on $(t, T)$. And as these observations about continuity apply also for the quantity $\mathbb{E}_{\mathbf{x}}\left[m_{j}(s, \beta+\sqrt{s-t} \mathbf{x})\right]$ itself, that the dispersion operator $D_{k}$ commutes under the time-integral in (6) follows by Leibniz's rule.

\section{Strong non-singularity}

Recall the definition of strong non-singularity in the main text.

Claim C.1 Let $\left\{f_{k}\right\}_{k \in \mathcal{K}}$ be a collection of continuous functions $f_{k}: \mathbb{R}^{L} \supseteq S \rightarrow \mathbb{R}^{K}$ (S open). For any $\mathbf{x}^{0} \in S$ the following are equivalent.

(i) The matrix $\left[f_{1}\left(\mathbf{x}^{0}\right) \cdots f_{K}\left(\mathbf{x}^{0}\right)\right]$ is non-singular.

(ii) There exists $\varepsilon>0$ such that $\left\{f_{k}\right\}_{k \in \mathcal{K}}$ satisfies strong non-singularity on $\mathcal{B}(\varepsilon)_{\mathbf{x}^{0}}^{K}$.

Proof We only need to show of course that (ii) implies (i). For any matrix $A:=\left[a_{n k}\right]_{(n, k) \in \mathcal{K} \times \mathcal{K}}$ and vector $\mathbf{v}:=\left(v_{1}, \ldots, v_{K}\right)^{\top}$, denote the maximal absolute value of their respective ele$\operatorname{ments}$ by $\max (A):=\max _{(n, k) \in \mathcal{K} \times \mathcal{K}}\left|a_{n k}\right|$ and $\max (\mathbf{v}):=\max _{k \in \mathcal{K}}\left|v_{k}\right|$. For any $n \in \mathcal{K}$ then we have

$$
\left|\mathbf{e}_{n}^{\top} A \mathbf{v}\right|=\left|\sum_{k \in \mathcal{K}} a_{n k} v_{k}\right| \leq \sum_{k \in \mathcal{K}}\left|a_{n k} v_{k}\right| \leq K \max (A) \max (\mathbf{v})
$$

where $\mathbf{e}_{n}$ denotes the $K$-dimensional column vector with one as its $n$th entry and zeroes everywhere else.

Suppose now that $\left[f_{1}\left(\mathbf{x}^{0}\right) \cdots f_{K}\left(\mathbf{x}^{0}\right)\right]$ is non-singular. Set $\delta_{0}=1 / \max \left(\left[f_{1}\left(\mathbf{x}^{0}\right) \cdots f_{K}\left(\mathbf{x}^{0}\right)\right]^{-1}\right)$ and take $\varepsilon_{0}>0$ such that $\left|f_{1}(\mathbf{x})-f_{1}\left(\mathbf{x}^{0}\right)\right|<\delta_{0} / K$ for all $\mathbf{x} \in \mathcal{B}_{\mathbf{x}^{0}}\left(\varepsilon_{0}\right) \subset S$. By the preceding observation, for any $\mathbf{x}^{1} \in \mathcal{B}_{\mathbf{x}^{0}}\left(\varepsilon_{0}\right)$, the matrix

$$
\left[f_{1}\left(\mathbf{x}^{1}\right) f_{2}\left(\mathbf{x}^{0}\right) \cdots f_{K}\left(\mathbf{x}^{0}\right)\right]=\left[f_{1}\left(\mathbf{x}^{0}\right) \cdots f_{K}\left(\mathbf{x}^{0}\right)\right]+\left(f_{1}\left(\mathbf{x}^{1}\right)-f_{1}\left(\mathbf{x}^{0}\right)\right) \mathbf{e}_{1}^{\top}
$$


is such that

$$
\left|\mathbf{e}_{1}^{\top}\left[f_{1}\left(\mathbf{x}^{0}\right) \cdots f_{K}\left(\mathbf{x}^{0}\right)\right]^{-1}\left(f_{1}\left(\mathbf{x}^{1}\right)-f_{1}\left(\mathbf{x}^{0}\right)\right)\right|<1
$$

That $\left[f_{1}\left(\mathbf{x}^{1}\right) f_{2}\left(\mathbf{x}^{0}\right) \cdots f_{K}\left(\mathbf{x}^{0}\right)\right]$ is non-singular at any $\mathbf{x}^{1} \in \mathcal{B}_{\mathbf{x}^{0}}\left(\varepsilon_{0}\right)$ follows from the matrix determinant lemma. ${ }^{24}$

Take now $\varepsilon_{1} \in\left(0, \varepsilon_{0}\right)$ and let $\operatorname{cl}\left(\mathcal{B}_{\mathbf{x}^{0}}\left(\varepsilon_{1}\right)\right)$ be the closure of $\mathcal{B}_{\mathbf{x}^{0}}\left(\varepsilon_{1}\right)$. By the continuity of the matrix inverse, the quantity

$$
\delta_{1}=\max _{\mathbf{x}^{1} \in \operatorname{cl}\left(\mathcal{B}_{\mathbf{x}^{0}}\left(\varepsilon_{1}\right)\right)} \max \left(\left[f_{1}\left(\mathbf{x}^{1}\right) \cdots f_{K}\left(\mathbf{x}^{0}\right)\right]^{-1}\right)
$$

is well defined. Let now $\varepsilon_{2}>0$ be such that $\left|f_{2}(\mathbf{x})-f_{2}\left(\mathbf{x}^{0}\right)\right|<1 /\left(K \delta_{1}\right)$ for all $\mathbf{x} \in$ $\mathcal{B}_{\mathbf{x}^{0}}\left(\varepsilon_{2}\right) \subset S$. By the previous argument, the matrix $\left[f_{1}\left(\mathbf{x}^{1}\right) f_{2}\left(\mathbf{x}^{2}\right) f_{3}\left(\mathbf{x}^{0}\right) \cdots f_{K}\left(\mathbf{x}^{0}\right)\right]$ is non-singular at any $\left(\mathbf{x}^{1}, \mathbf{x}^{2}\right) \in \mathcal{B}_{\mathbf{x}^{0}}\left(\min \left\{\varepsilon_{1}, \varepsilon_{2}\right\}\right)$. Successive iterations of this argument establish the existence of $\varepsilon_{3}, \ldots, \varepsilon_{K} \in \mathbb{R}_{++}$so that the matrix $\left[f_{1}\left(\mathbf{x}^{1}\right) \cdots f_{K}\left(\mathbf{x}^{K}\right)\right]$ is non-singular for any $\left(\mathbf{x}^{1}, \ldots, \mathbf{x}^{K}\right) \in \mathcal{B}_{\mathbf{x}^{0}}\left(\min \left\{\varepsilon_{1}, \ldots, \varepsilon_{K}\right\}\right)^{K}$. The claim follows by letting $\varepsilon:=\min \left\{\varepsilon_{1}, \ldots, \varepsilon_{K}\right\}$.

\section{References}

1. Anderson, R., Raimondo, R.: Market clearing and derivative pricing. Econ. Theory 25, 21-34 (2005)

2. Anderson, R., Raimondo, R.: Equilibrium in continuous-time financial markets: endogenously dynamically complete markets. Econometrica 71, 841-907 (2008)

3. Basak, S., Cuoco, D.: An equilibiurm model with restricted stock market participation. Rev. Financ. Stud. 11, 309-41 (1998)

4. Bick, A.: On the consistency of the Black-Scholes model with a general equilibrium framework. J. Financ. Quant. Anal. 22, 259-275 (1987)

5. Bick, A.: On viable diffusion price processes of the market portfolio. J. Finance 45, 673-89 (1990)

6. Bingham, N., Kiesel, R.: Risk-Neutral Valuation: Pricing and Hedging of Financial Derivatives. Springer, Berlin (2004)

7. Cochrane, J., Longstaff, F., Santa-Clara, P.: Two trees. Rev. Financ. Stud. 21, 347-85 (2008)

8. Cox Jr., J.I., Ross, S.: An intertemporal general equilibrium model of asset prices. Econometrica 53, 363-84 (1985)

9. Cuoco, D., He, H.: Dynamic aggregation and computation of equilibria in finite-dimensional economies with incomplete financial markets. Ann. Econ. Financ. 2, 265-96 (2001)

10. Detemple, J., Zapatero, F.: Asset prices in an exchange economy with habit formation. Econometrica 59, 1633-57 (1991)

11. Duffie, D.: Dynamic Asset Pricing Theory. Princeton University Press, Princeton (2001)

12. Duffie, D., Zaime, W.: The consumption-based CAPM. Econometrica 57, 1279-97 (1989)

13. Dumas, B.: Two-person dynamic equilibrium in a capital market. Rev. Financ. Stud. 2, 157-88 (1989)

14. Ehling, P., Heyerdahl-Larsen, C.: Complete and incomplete financial markets in multi-good economies. J. Econ. Theory 160, 438-62 (2015)

15. Farhi, E., Panageas, S.: Saving and investing for early retirement: a theoretical analysis. J. Financ. Econ. 83, 87-121 (2007)

16. He, H., Leland, H.: On equilibrium asset price processes. Rev. Financ. Stud. 6, 593-617 (1993)

17. Hugonnier, J.S.M.S., Trubowitz, E.: Endogenous completeness of diffusion driven equilibrium markets. Econometrica 80, 1249-70 (2012)

18. Karatzas, I., Lehoczky, J., Shreve, S.: Existence and uniquence of multi-agent equilibrium in a stochastic dynamic consumption/investement model. Math. Oper. Res. 15, 80-128 (1990)

19. Kramkov, D.: Existence of an endogenously complete equilibrium driven by a diffusion. Finance Stochast. 19, 1-22 (2015)

24 Let $A$ be a $K \times K$ matrix and $\mathbf{u}, \mathbf{v} K$-dimensional column vectors. The matrix determinant lemma states that, if $A$ is invertible then $\left|A+\mathbf{u} \times \mathbf{v}^{\top}\right|=\left|1+\mathbf{v}^{\top} A^{-1} \mathbf{u}\right| \times|A|$. 
20. Krantz, S., Parks, H.: A Primer of Real Analytic Functions, 2nd edn. Birkhauser Advanced Texts, Basler (2002)

21. Lucas, R.: Asset prices in an exchange economy. Econometrica 46, 1429-45 (1978)

22. Martin, I.: The Lucas Orchard. Econometrica 81, 55-111 (2013)

23. Merton, R.: Continuous-Time Finance. Basil Blackwell, Cambridge (1992)

24. Nielsen, L.: Pricing and Hedging of Derivative Securities. Oxford University Press, Oxford (1999)

25. Raimondo, R.: Market clearing, utility functions and security prices. Econ. Theory 25, $265-85$ (2005)

26. Riedel, F.: Existence of Arrow-Radner equilibrium with endogenously complete markets under incomplete information. J. Econ. Theory 97, 109-22 (2001)

27. Riedel, F., Herzberg, F.: Existence of Financial Equilibria in Continuous Time with Potentially Complete Markets. Working Papers, Institute of Mathematical Economics, Vol. 443, Bielefeld: Center for Mathematical Economics (2010). Available at https://pub.uni-bielefeld.de/record/2909312

28. Riedel, F., Herzberg, F.: Existence of financial equilibria in continuous time with potentially complete markets. J. Math. Econ. 49, 398-404 (2013)

29. Schilling, R.: Measures, Integrals, and Martingales. Cambridge University Press, Cambridge (2011)

30. Wang, S.: The integrability problem of asset prices. J. Econ. Theory 59, 199-213 (1993)

Publisher's Note Springer Nature remains neutral with regard to jurisdictional claims in published maps and institutional affiliations. 